Archive for

Organic Chemistry

Arkivoc 2020, part v, 161-175

\title{
Synthesis of some new 5-arylidene-2,4-thiazolidinedione esters
}

\author{
Ndivhuwo R. Tshiluka, Mpelegeng V. Bvumbi, Isaiah I. Ramaite, and Simon S. Mnyakeni-Moleele* \\ Department of Chemistry, University of Venda, Thohoyandou, Private Bag X5050, 0950 \\ Email: Mnyakeni.Moleele@univen.ac.za
}

Received 10-14-2020

Accepted 01-23-2021

Published on line 01-31-2021

\section{Abstract}

Compounds containing the 1,3-thiazolidine-2,4-dione scaffold are gaining increasing scientific interest as potential interventional agents for a variety of disease states. A four-step synthesis of ethyl-(2-(5-arylidine-2,4dioxothiazolidin-3-yl)acetyl)glycinates, alaninates, butanoates, valinates and norvalinates is described. The synthesis began by converting 1,3-thiazolidine-2,4-dione into its potassium salt, which was treated with ethyl (2-chloroacetamido)glycinates, alaninates, butanoates, valinates and norvalinates, respectively, to obtain the penultimate products. These products were then subjected to a Knoevenagel condensation reaction with different aldehydes to obtain the desired products in low to excellent yields.<smiles>[R]C(=O)[I-][I-]</smiles>

EtOH, Piperidine Reflux, 10-12 hours

30 examples $(4-98 \%)$<smiles>[R]C=C1SC(=O)N(CC(=O)NC([R])C(=O)OCC)C1=O</smiles>

Keywords: Thiazolidinedione, alaninate, glycinate, butanoate, valinate, norvalinate 


\section{Introduction}

1,3-Thiazolidine-2,4-dione (TZD)-containing compounds have been reported to show many different pharmacological activities. ${ }^{1} \mathrm{~A}$ few of these compounds are indicated in Figure 1. Glitazones, like rosiglitazone (1), are currently marketed for the treatment of type 2 diabetes. ${ }^{2}$ Thiazolidinedione, linked to chromones via benzylidene moiety (2), have been reported to act as aldose reductase inhibitors. ${ }^{3}$ Aneja and co-workers have reported that a series compounds of 5-((3-(aryl)-1-phenyl-1H-pyrazol-4-yl) methylene)-3-phenyl thiazolidine2,4-diones (3) have antibacterial and antiviral activity. ${ }^{4} \mathrm{~A}$ new series of 5 -benzylidene-2,4-thiazolidinedione derivatives (4) have been reported by Patil et al. to have anticancer activity. ${ }^{5}$ Rekha et al. synthesized 5substituted-arylidine-2,4-thiazolidinediones derivatives (5), and reported them to have anti-inflammatory activities. ${ }^{6}$ Sharma et al. have synthesized a series of 2,4 thiazolidinediones (6), following a structure-based virtual screening, in order to explore structure-activity relationships for inhibition of the $P$. falciparum cysteine protease falcipain-2 (FP-2) and whole-cell antiparasitic activity. ${ }^{7}$ Most compounds were reported to display low micromolar antiplasmodial activities against the P. falciparum drug-resistant W2 strain. ${ }^{8}$ Alagawadi and Alegaon ${ }^{9}$ reported the synthesis and in vitro antifungal activity of 5 -substituted-2,4-thiazolidinedione derivatives (7). Their compounds were found to be active against tested fungal strains at 1-64 $\mu \mathrm{g} / \mathrm{mL}$ concentrations. Kumar et al. have reported a series of 5-((E)-4-((E)-(substituted aryl/alkyl)methyl) benzylidene)thiazolidine-2,4-dione derivatives (8) which were tested for their antioxidant properties by determining their effects on superoxide anion formation and the 2,2-diphenyl-1-picrylhydrazyl (DPPH) stable free radical. ${ }^{10}$
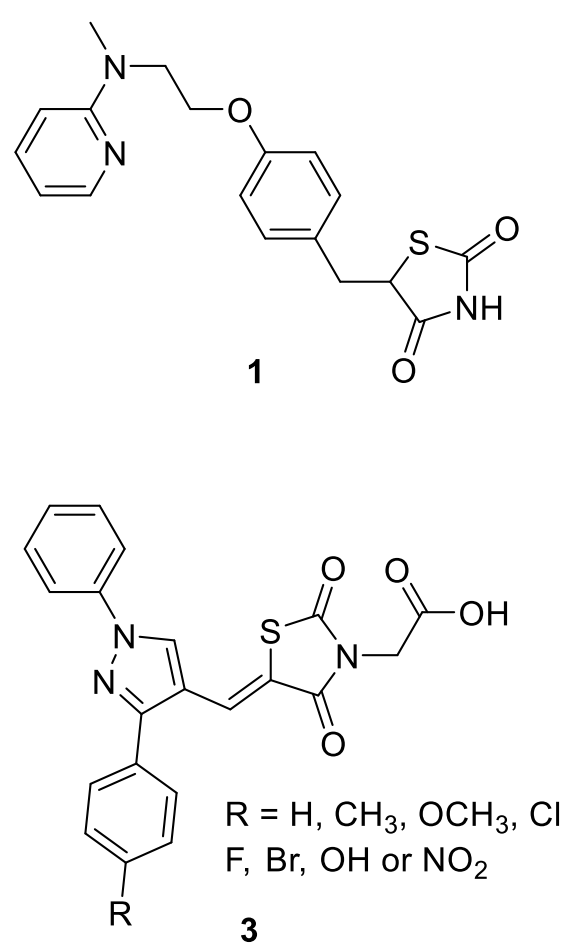<smiles>[R]c1cc([R1])c2c(=O)cc(-c3ccc(/C=C4\SC(=O)N(CC(=O)O)C4=O)cc3)oc2c1</smiles>

$\mathrm{R}=\mathrm{H}, \mathrm{CH}_{3}, \mathrm{NO}_{2}$ or $\mathrm{CH}\left(\mathrm{CH}_{3}\right)_{2}$

$\mathrm{R}^{1}=\mathrm{H}$ or $\mathrm{CH}_{3}$

2<smiles>O=C(COc1ccc(/C=C2\SC(=O)NC2=O)cc1)Nc1ccccn1</smiles> 


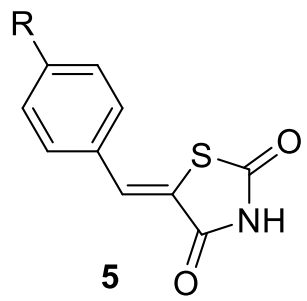

$\mathrm{R}=\mathrm{H}$, phenol, morpholine or aniline

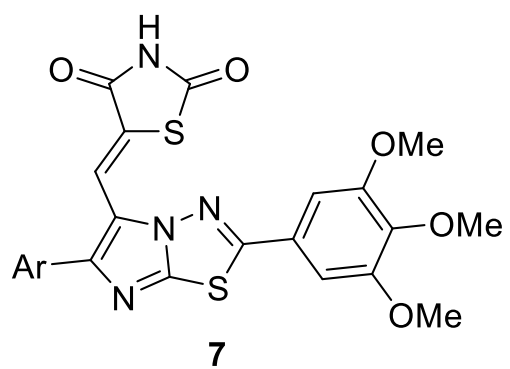

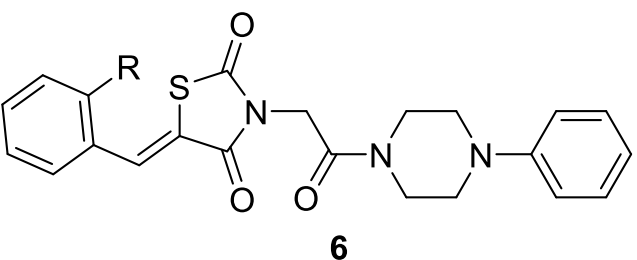

$\mathrm{R}=\mathrm{F}, \mathrm{Br}, \mathrm{Cl}$ or $\mathrm{CF}_{3}$

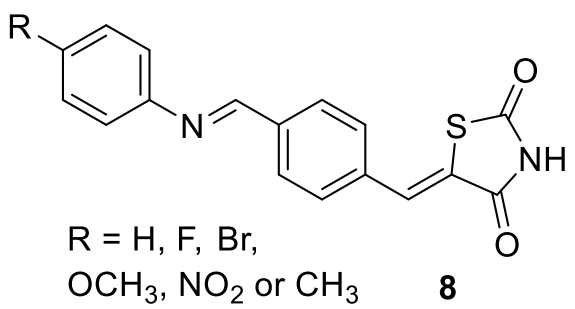

Figure 1. Compounds containing the thiazolidinedione moiety.

In view of the number of compounds containing the thiazolidinedione moiety possessing different biological activities shown above, we sought to synthesize novel 5-arylidene-2,4-thiazolidinedione esters. The syntheses of these compounds, using Knoevenagel condensation reaction as a key step, was designed schematically to consist of the arylidene (aryl) chain, TZD scaffold, two-carbon linker as well as an amino acid chain (Figure 2), which is similar to that described by Kumar et al. ${ }^{11}$

Two carbon linker

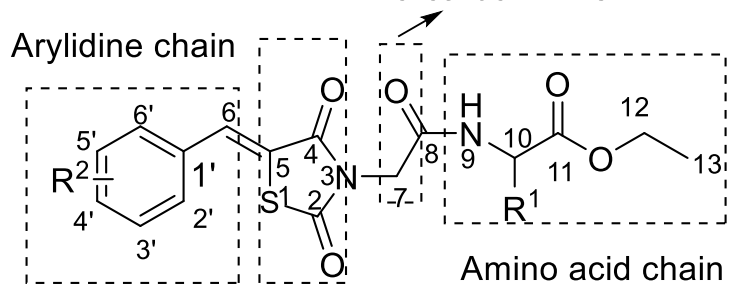

TZD scaffold

Figure 2. Schematic representation of ethyl-(2-(5-Arylidine-2,4-dioxothiazolidin-3-yl)acetyl)esters derivatives.

\section{Results and Discussion}

The synthesis of 5-arylidene-2,4-thiazolidinedione esters started with protection of the five different amino acids 9 as esters 10 by using thionyl chloride in the presence of ethanol at lower temperatures $\left(0-5{ }^{\circ} \mathrm{C}\right)$ (Scheme 1). Compounds 10 were then each treated with bromoacetyl chloride to obtain the ethyl (2chloroacetamido) esters 11. Treatment of compounds $\mathbf{1 1}$ with a potassium salt of thiazolidinedione ${ }^{12}$ gave compounds 12 in excellent yields (i.e., 12a, 89\%; 12b, 92\%; 12c, 88\%; 12d, 89\%; and 12e, 79\%). Finally, compounds 12 were subjected to Knoevenagel-reaction conditions using various aldehydes to provide the desired products 13 in low-to-excellent yields, as shown in Table 1 below. As the protected amino acid chain was extended from glycine to alanine, valine or norvaline (Scheme 1), a chiral centre was created at position 
$\mathrm{H}-10$ of the desired molecule (Figure 2). Thus, ${ }^{1} \mathrm{H}$ NMR spectra containing these amino acids moieties showed the multiplicity of the $\mathrm{N}-\mathrm{H}$ signal $\mathrm{H}-9$ as a doublet, and not a triplet that was shown by glycine-moietycontaining compounds. After a Knoevenagel condensation reaction step, ${ }^{1} \mathrm{H}$ NMR spectra of compounds $13 a-e$ were characterised by the absence of aldehydic proton peaks at $\approx 9 \mathrm{ppm}$, confirming the consumption of the aldehydes. In addition, the spectra were characterised by the appearance of an aryl proton peak as a singlet at $\sim 7.9$ ppm. ${ }^{13} \mathrm{C}$ NMR spectra of compounds 13a-e were characterised by lack of an aldehydic carbon peak at $\sim$ $190 \mathrm{ppm}$ with the appearance of a new aryl carbon signal at $\sim 134 \mathrm{ppm}$, confirming that the condensation reaction was successful. After the introduction of a fluorine atom at the para position of the phenyl ring, interestingly, the ${ }^{1} \mathrm{H}$ NMR spectra of compounds 13avii, 13bvii, 13dv, and 13eiv showed a coupling between the fluorine and the neighbouring proton signal $\mathrm{H}-3^{\prime}, \mathrm{H}-5^{\prime}$ at $7.81 \mathrm{ppm}$, and $\mathrm{H}-2^{\prime}, \mathrm{H}-6^{\prime}$ at 7.48 ppm appearing as a doublet of doublets instead of the expected doublet. This observation was further supported by ${ }^{13} \mathrm{C}$ NMR spectra which revealed coupling between the fluorine and the carbon signals C-2', C-6' as well as C- $3^{\prime}, \mathrm{C}^{\prime}-5^{\prime}$ which showed two extra signals appearing as doublets instead of the expected singlet. This was further confirmed by ${ }^{19} \mathrm{~F}$ NMR spectra of compounds 13avii, 13bvii, 13dv, and 13eiv, which showed coupling between protons $\mathrm{H}-3^{\prime}, \mathrm{H}-5^{\prime}, \mathrm{H}-2^{\prime}$ and $\mathrm{H}-6^{\prime}$ and appeared as a multiplet instead of a singlet.

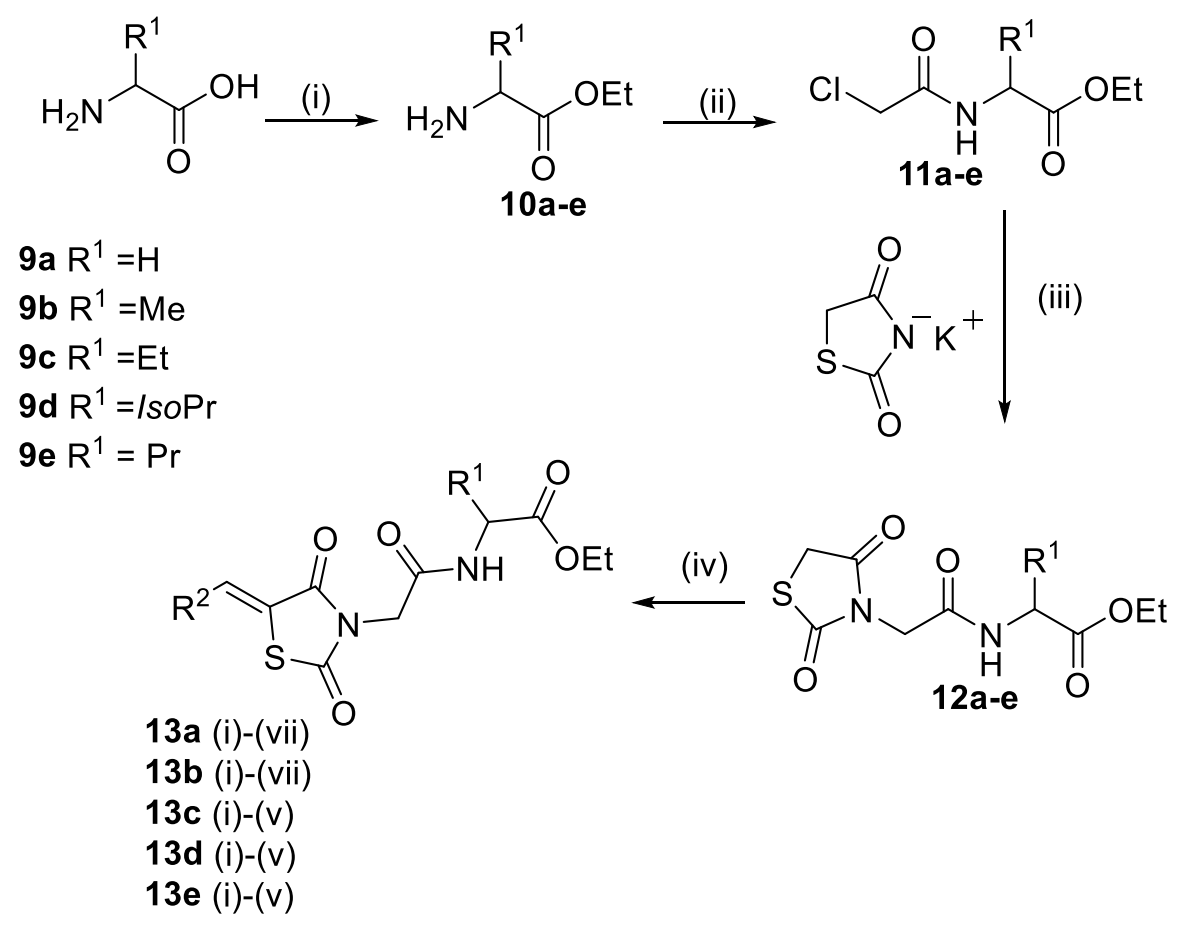

Scheme 1. Reagents and conditions: (i) $\mathrm{SOCl}_{2}$, ethanol, $0-5^{\circ} \mathrm{C}$; (ii) $\mathrm{K}_{2} \mathrm{CO}_{3}$, bromoacetyl chloride, $\mathrm{DCM},-10{ }^{\circ} \mathrm{C}$ to rt; (iii) THF, reflux; (iv) aldehydes, piperidine, ethanol, reflux. 
Table 1. \% yields of products 13

\begin{tabular}{|c|c|c|c|}
\hline Entry & $\mathrm{R}^{1}$ & $\mathrm{R}^{2}$ & Yield (\%) \\
\hline 13ai & \multirow{7}{*}{$\mathrm{H}$} & $\mathrm{Ph}$ & 50 \\
\hline 13aii & & 4-OMePh & 54 \\
\hline 13aiii & & $4-\mathrm{NO}_{2} \mathrm{Ph}$ & 98 \\
\hline 13aiv & & 4-OMe, 3-OHPh & 97 \\
\hline $13 a v$ & & piperonayl & 54 \\
\hline 13avi & & 4-MePh & 46 \\
\hline 13avii & & 4-FPh & 12 \\
\hline $13 b i$ & \multirow{8}{*}{ Me } & $\mathrm{Ph}$ & 50 \\
\hline 13bii & & 4-OMePh & 98 \\
\hline 13biii & & $4-\mathrm{NO}_{2} \mathrm{Ph}$ & 90 \\
\hline 13biv & & 4-OMe, 3-OHPh & 61 \\
\hline $13 b v$ & & piperonayl & 53 \\
\hline 13bvi & & 4-MePh & 31 \\
\hline 13bvii & & 4-FPh & 11 \\
\hline 13bviii & & Furanyl & 34 \\
\hline $13 \mathrm{ci}$ & \multirow{5}{*}{ Et } & $\mathrm{Ph}$ & 10 \\
\hline $13 \mathrm{cii}$ & & 4-OMePh & 37 \\
\hline 13ciii & & 4-MePh & 8 \\
\hline 13 civ & & 4-OHPh & 6 \\
\hline $13 \mathrm{cv}$ & & Furanyl & 11 \\
\hline 13di & \multirow{5}{*}{ Iso-Pr } & $\mathrm{Ph}$ & 24 \\
\hline 13dii & & 4-OMePh & 38 \\
\hline 13diii & & 4-OMe, 3-OHPh & 12 \\
\hline 13div & & 4-OHPh & 4 \\
\hline $13 d v$ & & 4-FPh & 11 \\
\hline $13 e i$ & \multirow{5}{*}{$\operatorname{Pr}$} & 4-OMePh & 17 \\
\hline 13eii & & 4-MePh & 12 \\
\hline 13eiii & & 4-OHPh & 26 \\
\hline 13eiv & & 4-FPh & 21 \\
\hline $13 e v$ & & Furanyl & 65 \\
\hline
\end{tabular}

\section{Conclusions}

Five series of glycinates $\mathbf{1 3 a}$, alaninates $\mathbf{1 3 b}$, butanoates $\mathbf{1 3 c}$, valinates $\mathbf{1 3 d}$, and norvalinates $\mathbf{1 3 e}$ derivatives were successfully synthesised in average yields of $52 \%, 54 \%, 14 \%, 14 \%$ and $28 \%$, respectively, in four reaction steps. Modified known methods were followed in synthesizing these compounds. 


\section{Experimental Section}

General. All reagents used were analytical grade reagents from Sigma-Aldrich and Fluka. Thin-layer chromatography (TLC) was carried out using Macherey-Nagel Alugram Sil G/UV 254 plates, pre-coated with 0.25 $\mathrm{mm}$ silica gel 60. Detection was done under ultraviolet light at $254 \mathrm{~nm} .{ }^{1} \mathrm{H}$ NMR (400 MHz), ${ }^{13} \mathrm{C}$ NMR (100 $\mathrm{MHz}$ ) and ${ }^{19} \mathrm{~F}$ NMR (376.5 MHz) spectra were recorded on a Bruker $400 \mathrm{MHz}$ spectrometer using DMSO- $d_{6}$ or $\mathrm{CDCl}_{3}$ as solvents and TMS at $0.00 \mathrm{ppm}$ as an internal standard. Values for the chemical shifts are expressed in parts per million (ppm). The following abbreviations are used: br.s for broad singlet, $s$ for singlet, $d$ for doublets, dd for doublet of doublets, q for quartet, quint for quintet and $m$ for multiplet and $(J)$ for coupling constants measured in hertz $(\mathrm{Hz})$. All the melting points were determined on a Buchi melting point B-540 apparatus using capillary tubes. Infrared spectra were run on a Bruker platinum 22 vector Fourier Transform spectrometer (FTIR). Mass spectra (High Resolution) were recorded on a Waters GCT using a column called the Restek Rxi Wintegra Guard ( $15 \mathrm{~m}, 0.25 \mathrm{~mm}$ ID, $0.25 \mu \mathrm{m}$ film thickness) mass spectrometer. The samples were dissolved in acetonitrile and injected at a volume of $1 \mu \mathrm{l}$ at a mode of $10: 1$ and temperature of $280{ }^{\circ} \mathrm{C}$. The source temperature was $100{ }^{\circ} \mathrm{C}$ and the de-solvation temperature was set at $300{ }^{\circ} \mathrm{C}$. Helium gas was used as the carrier gas. The software used to control the hyphenated system and to do all data manipulation was MassLynx 4.1 (SCN 704).

\section{General procedure for synthesis of ethyl (2-chloroacetamido) esters (11a-e)}

Amino esters (10), which were obtained from corresponding acids (9) were cooled to $-10{ }^{\circ} \mathrm{C}$ before being treated with a solution of bromoacetyl chloride $(1 \mathrm{mmol})$ and potassium carbonate $(3 \mathrm{mmol})$ in a mixture of dichloromethane and water (1:1). The resulting solution was allowed to warm up to room temperature before been stirred for 16 hours at the same temperature. After this reaction time, the mixture was extracted with dichloromethane, and the organic extracts were subsequently washed with brine before being dried over $\mathrm{MgSO}_{4}$ and filtered before being concentrated on a rotary evaporator. The products obtained were used without further purification.

Ethyl (2-chloroacetyl)glycinate (11a). ${ }^{13}$ Compound was obtained as a white solid (4.90 g, 96\%); m.p = 75-76 ${ }^{\circ} \mathrm{C}$. Spectroscopic data were similar to the one described by Kumar et al.

Ethyl (2-chloroacetyl)alaninate (11b). ${ }^{14}$ Compound was obtained as a colourless oil (7.60 g, $98 \%$ ). Spectroscopic data were similar to those described by Aladzheva et al.

Ethyl 2-(2-chloroacetamido)butanoate (11c). A reaction of $10 \mathrm{c}^{15}$ (2.04 g, $\left.15.2 \mathrm{mmol}\right)$ and bromoacetyl chloride (1.27 ml, $15.2 \mathrm{mmol})$ gave compound $11 \mathrm{c}$ as a brown oil $(2.74 \mathrm{~g}, 88 \%)$; IR $\left(\mathrm{KBr} \mathrm{cm}^{-1}\right): 3233,2968$, 1727, 1250; ${ }^{1} \mathrm{H}$ NMR (400 MHz, $\left.\mathrm{CDCl}_{3}\right) \delta_{\mathrm{H}}(\mathrm{ppm}) 7.17$ (d, $\left.1 \mathrm{H}, J 6.8, \mathrm{~Hz}, \mathrm{NH}\right), 4.45$ (quint, $1 \mathrm{H}, J 4.0 \mathrm{~Hz}, \mathrm{CHNH}$ ), 4.12 (q, $\left.2 \mathrm{H}, J 4.0 \mathrm{~Hz}, \mathrm{OCH}_{2} \mathrm{CH}_{3}\right), 3.84\left(\mathrm{~s}, 2 \mathrm{H}, \mathrm{CH}_{2} \mathrm{Cl}\right), 1.81-1.91\left(1 \mathrm{H}, \mathrm{m}, \mathrm{CHCH}_{3}\right), 1.22\left(\mathrm{t}, 3 \mathrm{H}, J 6.0 \mathrm{~Hz}, \mathrm{CH}_{3} \mathrm{CH}_{2} \mathrm{O}\right)$, $0.87\left(3 \mathrm{H}, \mathrm{t}, J 7.2 \mathrm{~Hz}, \mathrm{CH}_{3} \mathrm{CH}_{2} \mathrm{O}\right) ;{ }^{13} \mathrm{C} \mathrm{NMR}\left(100 \mathrm{MHz}, \mathrm{CDCl}_{3}\right) \delta_{\mathrm{c}}(\mathrm{ppm}) 171.7(\mathrm{C}=\mathrm{O}) 165.9(\mathrm{C}=\mathrm{O}), 61.5\left(\mathrm{OCH}_{2} \mathrm{CH}_{3}\right)$, $42.4\left(\mathrm{CH}_{2} \mathrm{Cl}\right), 28.7(\mathrm{CHNH}), 25.3\left(\mathrm{CH}_{2} \mathrm{CH}_{3}\right), 14.1\left(\mathrm{CH}_{3} \mathrm{CH}_{2} \mathrm{O}\right) .9 .3\left(\mathrm{CH}_{3} \mathrm{CH}_{2}\right)$.

Ethyl (2-chloroacetyl)valinate (11d). ${ }^{16}$ Compound 11d was obtained as a colourless oil (6.74 $\left.\mathrm{g}, 89 \%\right)$. Spectroscopic data were similar to those described by Khadidja et al.

Ethyl (2-chloroacetyl)norvalinate (11e). A reaction of $10 \mathrm{e}^{15}(5.03 \mathrm{~g}, 34.4 \mathrm{mmol})$ and bromoacetyl chloride (2.89 ml, $34.4 \mathrm{mmol})$ gave compound 10e as a colourless oil (5.74 g, 79\%); IR (KBr cm$\left.{ }^{-1}\right): 3272,2962,1733$, 1290; ${ }^{1} \mathrm{H}$ NMR $\left(400 \mathrm{MHz}, \mathrm{CDCl}_{3}\right) \delta_{\mathrm{H}}(\mathrm{ppm}) 8.68(\mathrm{~d}, 1 \mathrm{H}, \mathrm{J} 3.6, \mathrm{~Hz}, \mathrm{NH}), 5.75\left(\mathrm{~s}, 2 \mathrm{H}, \mathrm{CH}_{2} \mathrm{Cl}\right) 4.22$ (quint, $1 \mathrm{H}, \mathrm{J} 4.8$ $\mathrm{Hz}, \mathrm{CHNH}), 4.13\left(\mathrm{q}, 2 \mathrm{H}, \mathrm{J} 4.0 \mathrm{~Hz}, \mathrm{OCH}_{2} \mathrm{CH}_{3}\right), 3.93-3.89\left(\mathrm{~m}, 2 \mathrm{H}, \mathrm{CH}_{2} \mathrm{CH}\right), 1.70-1.64\left(1 \mathrm{H}, \mathrm{m}, \mathrm{CH}_{2} \mathrm{CH}_{3}\right), 1.22(\mathrm{t}, 3 \mathrm{H}, J$ $\left.2.0 \mathrm{~Hz}, \mathrm{CH}_{3} \mathrm{CH}_{2} \mathrm{O}\right) ; 0.88$ (t, 3H, J $\left.7.2 \mathrm{~Hz}, \mathrm{CH}_{3} \mathrm{CH}_{2}\right) ;{ }^{13} \mathrm{C} \mathrm{NMR}\left(100 \mathrm{MHz}, \mathrm{CDCl}_{3}\right) \delta_{\mathrm{c}}(\mathrm{ppm}) 172.1$ (C=O) 166.7 (C=O), $62.1\left(\mathrm{OCH}_{2} \mathrm{CH}_{3}\right), 52.5(\mathrm{CHNH}), 33.4\left(\mathrm{CH}_{2} \mathrm{CH}_{3}\right), 29.3\left(\mathrm{CH}_{2} \mathrm{Cl}\right), 18.9\left(\mathrm{CH}_{3} \mathrm{CH}\right), 14.5\left(\mathrm{CH}_{3} \mathrm{CH}_{2} \mathrm{O}\right), 13.9\left(\mathrm{CH}_{3} \mathrm{CH}_{2}\right)$. 


\section{General procedure for synthesis of ethyl 2-(2,4-dioxothiazolidin-3-yl) acetyl esters (12a-e)}

A mixture of potassium salt of 1,3-thiazolidine-2,4-dione $(1 \mathrm{mmol})$ and ethyl (2-chloroacetamido) derivatives (11a-e) $(1 \mathrm{mmol})$ in tetrahydrofuran (THF) was refluxed for 12 hours. After cooling to room temperature, the mixture was filtered through cotton wool and the filtrate concentrated on a rotary evaporator to provide the ethyl 2-(2,4-dioxothiazolidin-3-yl) acetyl derivatives (12a-e) which were used without further purification.

Ethyl (2-(2,4-dioxothiazolidin-3-yl)acetyl)glycinate (12a). A reaction of the potassium salt of 1,3-thiazolidine2,4-dione (4.50 g, $38.3 \mathrm{mmol})$ and $11 \mathrm{a}(6.90 \mathrm{~g}, 38.3 \mathrm{mmol})$ gave compound $12 \mathrm{a}$ as a white solid (8.50 g, $89 \%)$; m.p = 110-112 ${ }^{\circ} \mathrm{C}$; IR (KBr cm $\left.{ }^{-1}\right): 3294,3108$ 1738, 1652; ${ }^{1} \mathrm{H}$ NMR (400 MHz, DMSO- $\left.d_{6}\right) \delta_{\mathrm{H}}(\mathrm{ppm}) 8.67(\mathrm{t}, 1 \mathrm{H}, J$ $5.6 \mathrm{~Hz}, \mathrm{NH}), 4.27\left(\mathrm{~s}, 2 \mathrm{H}, \mathrm{CH}_{2} \mathrm{~N}\right), 4.17\left(\mathrm{~s}, 2 \mathrm{H}, \mathrm{CH}_{2} \mathrm{~S}\right), 4.11\left(\mathrm{q}, 2 \mathrm{H}, J 7.2 \mathrm{~Hz}, \mathrm{OCH}_{2} \mathrm{CH}_{3}\right), 3.86\left(\mathrm{~d}, 2 \mathrm{H}, J 5.6 \mathrm{~Hz}, \mathrm{CH}_{2} \mathrm{NH}\right)$, 1.19 (t, 3H, J $\left.7.2 \mathrm{~Hz}, \mathrm{CH}_{3} \mathrm{CH}_{2} \mathrm{O}\right) ;{ }^{13} \mathrm{C} \mathrm{NMR}(100 \mathrm{MHz}$, DMSO-d $) \delta_{\mathrm{c}}(\mathrm{ppm}) 172.4$ (C=O), 172.0 (C=O), 169.9 (C=O), $166.2(\mathrm{C}=\mathrm{O}), 61.0\left(\mathrm{OCH}_{2} \mathrm{CH}_{3}\right), 43.4\left(\mathrm{CH}_{2} \mathrm{~N}\right), 41.5\left(\mathrm{CH}_{2} \mathrm{NH}\right), 34.5\left(\mathrm{CH}_{2} \mathrm{~S}\right), 14.5\left(\mathrm{CH}_{3} \mathrm{CH}_{2} \mathrm{O}\right)$.

Ethyl (2-(2,4-dioxothiazolidin-3-yl)acetyl)alaninate (12b). A reaction of the potassium salt of 1,3-thiazolidine2,4-dione (3.55 g, $30.3 \mathrm{mmol}$ ) and $\mathbf{1 1 b}$ (5.87 g, $30.3 \mathrm{mmol})$ gave compound $\mathbf{1 2 b}$ as a white solid (9.70 g, 92\%); m.p = 100-101 ${ }^{\circ} \mathrm{C} ; \quad I R\left(\mathrm{KBr} \mathrm{cm}^{-1}\right): 3288,3078,1735,1687 ;{ }^{1} \mathrm{H}$ NMR $\left(400 \mathrm{MHz}, \mathrm{DMSO}-d_{6}\right) \delta_{\mathrm{H}}(\mathrm{ppm}) 6.62(\mathrm{~d}, 1 \mathrm{H}, J$ $6.4 \mathrm{~Hz}, \mathrm{NH}$ ), 4.48 (quint, $1 \mathrm{H}, J 7.2 \mathrm{~Hz}, \mathrm{CHNH}$ ), $4.25\left(\mathrm{~s}, 2 \mathrm{H}, \mathrm{CH}_{2} \mathrm{~N}\right.$ ), 4.13 (q, 2H, J $\left.7.2 \mathrm{~Hz}, \mathrm{OCH}_{2} \mathrm{CH}_{3}\right), 3.99(\mathrm{~s}, 2 \mathrm{H}$, $\mathrm{CH}_{2} \mathrm{~S}$ ), 1.33 (d, $3 \mathrm{H}, J 7.2 \mathrm{~Hz}, \mathrm{CH}_{3} \mathrm{CH}$ ), 1.21 (t, 3H, J 7.2, $\mathrm{CH}_{3} \mathrm{CH}_{2} \mathrm{O}$ ); ${ }^{13} \mathrm{C}$ NMR (100 MHz, DMSO-d $\left.d_{6}\right), \delta_{c}$ (ppm) 172.7 $(\mathrm{C}=\mathrm{O}), 171.4(\mathrm{C}=\mathrm{O}), 171.1(\mathrm{C}=\mathrm{O}), 164.3(\mathrm{C}=\mathrm{O}), 61.7\left(\mathrm{OCH}_{2} \mathrm{CH}_{3}\right), 48.5(\mathrm{CHNH}), 43.5\left(\mathrm{CH}_{2} \mathrm{~N}\right), 33.9\left(\mathrm{CH}_{2} \mathrm{~S}\right), 18.3$ $\left(\mathrm{CH}_{3} \mathrm{CH}\right), 14,1\left(\mathrm{CH}_{3} \mathrm{CH}_{2} \mathrm{O}\right)$.

Ethyl 2-(2-(2,4-dioxothiazolidin-3-yl)acetamido)butanoate (12c). A reaction of the potassium salt of 1,3thiazolidine-2,4-dione $(4.50 \mathrm{~g}, 21.7 \mathrm{mmol})$ and $11 \mathrm{c}(2.54 \mathrm{~g}, 21.7 \mathrm{mmol})$ gave compound 12c as a white solid $(5.43 \mathrm{~g}, 79 \%) ; \mathrm{m} . \mathrm{p}=109-110^{\circ} \mathrm{C}$; IR $\left(\mathrm{KBr} \mathrm{cm}^{-1}\right): 3279,3077,1738,1682,{ }^{1} \mathrm{H} \mathrm{NMR}(400 \mathrm{MHz}$, DMSO-d $) \delta_{\mathrm{H}}(\mathrm{ppm})$ 6.55 (d, $1 \mathrm{H}, J 6.4 \mathrm{~Hz}, \mathrm{NH}$ ), 4.35 (quint, $1 \mathrm{H}, J 7.2 \mathrm{~Hz}, \mathrm{CHNH}$ ), 4.24 (s, $2 \mathrm{H}, \mathrm{CH}_{2} \mathrm{NH}$ ), 4.23 (q, $2 \mathrm{H}, J 4.0 \mathrm{~Hz}, \mathrm{OCH}_{2} \mathrm{CH}_{3}$ ), $4.06\left(\mathrm{~s}, 2 \mathrm{H}, \mathrm{CH}_{2} \mathrm{~S}\right), 1.94-1.86\left(\mathrm{~m}, 1 \mathrm{H}, \mathrm{CH}_{a} \mathrm{CH}_{3}\right), 1.79-1.68\left(\mathrm{~m}, 1 \mathrm{H}, \mathrm{CH}_{b} \mathrm{CH}_{3}\right), 1.30\left(\mathrm{t}, 3 \mathrm{H}, J 8.0 \mathrm{~Hz}, \mathrm{CH}_{3} \mathrm{CH}_{2}\right), 0.91(\mathrm{t}$, $3 \mathrm{H}$, J $\left.7.6 \mathrm{~Hz}, \mathrm{CH}_{3} \mathrm{CH}_{2} \mathrm{O}\right) ;{ }^{13} \mathrm{C}$ NMR (100 MHz, DMSO-d $)$, $\delta_{c}(\mathrm{ppm}) 172.1(\mathrm{C}=\mathrm{O}), 171.5(\mathrm{C}=\mathrm{O}), 171.0(\mathrm{C}=\mathrm{O}), 165.9$ $(\mathrm{C}=\mathrm{O}), 60.9\left(\mathrm{OCH}_{2} \mathrm{CH}_{3}\right), 58.0(\mathrm{CHNH}), 43.3\left(\mathrm{CH}_{2} \mathrm{~N}\right), 34.4\left(\mathrm{CH}_{2} \mathrm{~S}\right), 30.6\left(\mathrm{CH}_{2} \mathrm{CH}_{3}\right), 19.3\left(\mathrm{CH}_{3} \mathrm{CH}\right), 18.5\left(\mathrm{CH}_{3} \mathrm{CH}_{2}\right), 14.5$ $\left(\mathrm{CH}_{3} \mathrm{CH}_{2} \mathrm{O}\right)$.

Ethyl (2-(2,4-dioxothiazolidin-3-yl)acetyl)valinate (12d). A reaction of the potassium salt of 1,3-thiazolidine2,4-dione ( $3.79 \mathrm{~g}, 32.3 \mathrm{mmol}$ ) and $\mathbf{1 1 d}(7.16 \mathrm{~g}, 32.3 \mathrm{mmol})$ gave compound $\mathbf{1 2 d}$ as a white solid (7.91 g, 81\%); $\mathrm{m} . \mathrm{p}=118-120^{\circ} \mathrm{C} ; \mathrm{IR}\left(\mathrm{KBr} \mathrm{cm}{ }^{-1}\right): 3262,3080,1736,1685 ;{ }^{1} \mathrm{H} \mathrm{NMR}\left(400 \mathrm{MHz}, \mathrm{DMSO}-d_{6}\right) \delta_{\mathrm{H}}(\mathrm{ppm}) 8.57(\mathrm{~d}, 1 \mathrm{H}, \mathrm{J}$ $7.6 \mathrm{~Hz}, \mathrm{NH}$ ), $4.28\left(\mathrm{~s}, 2 \mathrm{H}, \mathrm{CH}_{2} \mathrm{~N}\right.$ ), 4.16 (quint, $\left.1 \mathrm{H}, J 8.4 \mathrm{~Hz}, \mathrm{CHNH}\right), 4.12\left(\mathrm{q}, 2 \mathrm{H}, J 4.0 \mathrm{~Hz}, \mathrm{OCH}_{2} \mathrm{CH}_{3}\right), 4.09(\mathrm{~s}, 2 \mathrm{H}$, $\left.\mathrm{CH}_{2} \mathrm{~S}\right), 2.04-2.03\left(\mathrm{~m}, 2 \mathrm{H}, \mathrm{CH}\left(\mathrm{CH}_{3}\right)_{2}\right), 1.19\left(\mathrm{t}, 3 \mathrm{H}, \mathrm{J} 6.4 \mathrm{~Hz}, \mathrm{CH}_{3} \mathrm{CH}_{2} \mathrm{O}\right), 0.87\left(\mathrm{~s}, 6 \mathrm{H},\left(\mathrm{CH}_{3}\right)_{2} \mathrm{CH}\right) ;{ }^{13} \mathrm{C} \mathrm{NMR}(100 \mathrm{MHz}$, DMSO- $\left.d_{6}\right), \delta_{c}(\mathrm{ppm}) 172.3(\mathrm{C}=\mathrm{O}), 171.9(\mathrm{C}=\mathrm{O}), 171.6(\mathrm{C}=\mathrm{O}), 165.9(\mathrm{C}=\mathrm{O}), 61.0\left(\mathrm{OCH}_{2} \mathrm{CH}_{3}\right), 58.0(\mathrm{CHNH}), 43.3$ $\left(\mathrm{CH}_{2} \mathrm{~N}\right), 34.3\left(\mathrm{CH}_{2} \mathrm{~S}\right), 30.6\left(\mathrm{CH}\left(\mathrm{CH}_{3}\right)_{2}\right), 19.6\left(\mathrm{CH}_{3} \mathrm{CH}\right), 18.5\left(\mathrm{CH}_{3} \mathrm{CH}\right), 14.6\left(\mathrm{CH}_{3} \mathrm{CH}_{2} \mathrm{O}\right)$.

Ethyl (2-(2,4-dioxothiazolidin-3-yl)acetyl)norvalinate (12e). A reaction of the potassium salt of 1,3thiazolidine-2,4-dione $(3.97 \mathrm{~g}, 33.8 \mathrm{mmol})$ and $11 \mathrm{e}(7.50 \mathrm{~g}, 33.8 \mathrm{mmol})$ gave compound $12 \mathrm{e}$ as a white solid $(8.08 \mathrm{~g}, 81 \%) ; \mathrm{m} . \mathrm{p}=119-120^{\circ} \mathrm{C} ;$; IR $\left(\mathrm{KBr} \mathrm{cm}^{-1}\right): 3282,3076,1737,1685 ;{ }^{1} \mathrm{H} \mathrm{NMR}\left(400 \mathrm{MHz}, \mathrm{DMSO}-d_{6}\right) \delta_{\mathrm{H}}(\mathrm{ppm})$ $8.62(\mathrm{~d}, 1 \mathrm{H}, \mathrm{J} 6.4 \mathrm{~Hz}, \mathrm{NH}), 4.27\left(\mathrm{~s}, 2 \mathrm{H}, \mathrm{CH}_{2} \mathrm{~N}\right.$ ), 4.22 (quint, $\left.1 \mathrm{H}, \mathrm{J} 8.0 \mathrm{~Hz}, \mathrm{CHNH}\right), 4.17\left(\mathrm{~s}, 2 \mathrm{H}, \mathrm{CH}_{2} \mathrm{~S}\right), 4.08(\mathrm{q}, 2 \mathrm{H}, \mathrm{J}$ $\left.5.6 \mathrm{~Hz}, \mathrm{OCH}_{2} \mathrm{CH}_{3}\right), 1.65-1.59\left(\mathrm{~m}, 2 \mathrm{H}, \mathrm{CH}_{2} \mathrm{CH}\right), 1.31-1.19\left(\mathrm{~m}, 2 \mathrm{H}, \mathrm{CH}_{2} \mathrm{CH}_{3}\right), 1.18\left(\mathrm{t}, 3 \mathrm{H}, \mathrm{J} 6.4 \mathrm{~Hz}, \mathrm{CH}_{3} \mathrm{CH}_{2} \mathrm{O}\right), 0.87(\mathrm{t}$, $\left.3 \mathrm{H}, J 6.4 \mathrm{~Hz}, \mathrm{CH}_{3} \mathrm{CH}_{2}\right) ;{ }^{13} \mathrm{C}$ NMR (100 MHz, DMSO-d $\left.)_{6}\right), \delta_{\mathrm{C}}(\mathrm{ppm}) 172.3(\mathrm{C}=\mathrm{O}), 172.2(\mathrm{C}=\mathrm{O}), 172.0(\mathrm{C}=\mathrm{O}), 165.8$ $(\mathrm{C}=\mathrm{O}), 61.0\left(\mathrm{OCH}_{2} \mathrm{CH}_{3}\right), 52.4(\mathrm{CHNH}), 43.3\left(\mathrm{CH}_{2} \mathrm{~N}\right), 34.4\left(\mathrm{CH}_{2} \mathrm{~S}\right), 33.5\left(\mathrm{CH}_{2} \mathrm{CH}\right), 18.9\left(\mathrm{CH}_{2} \mathrm{CH}_{3}\right), 14.5\left(\mathrm{CH}_{3} \mathrm{CH}_{2} \mathrm{O}\right)$, $13.9\left(\mathrm{CH}_{3} \mathrm{CH}_{2}\right)$.

General procedure for the synthesis of ethyl (2-(5-arylidene-2,4-dioxothiazolidin-3-yl)acetyl)ester derivatives (13a-e). To a suspension of compounds $12(1 \mathrm{mmol})$ in ethanol was added appropriate aldehydes 
(1 mmol) followed by catalytic amount of piperidine (10\%). The resultant mixture was refluxed for 12 hours. After being allowed to cool down to room temperature, the mixture was poured into ice water and the resultant precipitate collected by filtration. The solids obtained were further purified by washing with methanol to afford the desired compounds (13a-e).

Ethyl (2-(5-benzylidene-2,4-dioxothiazolidin-3-yl)acetyl)glycinate (13ai). A reaction of benzaldehyde $(0.40 \mathrm{ml}$, $3.84 \mathrm{mmol}$ ) and 12a (1.03 g, $3.84 \mathrm{mmol})$ gave compound 13ai as a brown solid (0.71 g, 52\%); m.p = 185-187 ${ }^{\circ} \mathrm{C}$; IR $\left(\mathrm{KBr} \mathrm{cm}^{-1}\right)$ : 3210, 3076, 1745, 1737, 1724, 1684, 1186; ${ }^{1} \mathrm{H}$ NMR $\left(400 \mathrm{MHz}, \mathrm{DMSO}-d_{6}\right) \delta_{\mathrm{H}}(\mathrm{ppm}) 8.78(\mathrm{t}, 1 \mathrm{H}$, $J=5.6 \mathrm{~Hz}, \mathrm{NH}), 7.97(\mathrm{~s}, 1 \mathrm{H}, \operatorname{Aryl}-\mathrm{H}), 7.66(\mathrm{~d}, 2 \mathrm{H}, J 6.8 \mathrm{~Hz}, 2 \times \operatorname{Ar}-\mathrm{H}), 7.56-7.53(\mathrm{~m}, 3 \mathrm{H}, 3 \times \operatorname{Ar}-\mathrm{H}), 4.35(\mathrm{~s}, 2 \mathrm{H}$, $\mathrm{CH}_{2} \mathrm{~N}$ ), 4.09 (q, $2 \mathrm{H}, J 6.8 \mathrm{~Hz}, \mathrm{OCH}_{2} \mathrm{CH}_{3}$ ), 3.89 (d, $\left.2 \mathrm{H}, J 4.8 \mathrm{~Hz}, \mathrm{CH}_{2} \mathrm{NH}\right), 1.20\left(\mathrm{t}, 3 \mathrm{H}, J 6.8 \mathrm{~Hz}, \mathrm{CH}_{3} \mathrm{CH}_{2} \mathrm{O}\right) ;{ }^{13} \mathrm{C} \mathrm{NMR}$ $\left(100 \mathrm{MHz}, \mathrm{DMSO}-d_{6}\right), \delta_{c}(\mathrm{ppm}) 169.9(\mathrm{C}=0), 167.5(\mathrm{C}=\mathrm{O}, 166.6(\mathrm{C}=\mathrm{O}), 165.7(\mathrm{C}=\mathrm{O}), 133.8(\operatorname{Aryl}-\mathrm{CH}), 133.3(\mathrm{Ar}-$ $\mathrm{CH}), 131.2(\mathrm{Ar}-\mathrm{C}), 130.6(2 \times \mathrm{Ar}-\mathrm{CH}), 129.9(2 \times \mathrm{Ar}-\mathrm{CH}), 121.6(\mathrm{Ar}-\mathrm{C}), 61.0\left(\mathrm{OCH}_{2} \mathrm{CH} 3\right), 43.7\left(\mathrm{CH}_{2} \mathrm{~N}\right), 41.3$ $\left(\mathrm{CH}_{2} \mathrm{NH}\right), 14.49\left(\mathrm{CH}_{3} \mathrm{CH}_{2} \mathrm{O}\right)$; HRMS (ESI-TOF) $\mathrm{m} / \mathrm{z}$; calculated for $\mathrm{C}_{16} \mathrm{H}_{16} \mathrm{~N}_{2} \mathrm{O}_{5} \mathrm{~S}-\mathrm{H}$ : 347.0780, found $\mathrm{M}^{+}-\mathrm{H}$ : 347.0783.

Ethyl (2-(5-(4-methoxybenzylidene)-2,4-dioxothiazolidin-3-yl)acetyl)glycinate (13aii). ${ }^{11}$ A reaction of $p$ anisaldehyde $(0.47 \mathrm{ml}, 3.84 \mathrm{mmol})$ and $12 \mathrm{a}(1.05 \mathrm{~g}, 3.84 \mathrm{mmol})$ gave compound 13aii as a white solid (0.72 $\mathrm{g}$, $50 \%) ; m . p=208-211^{\circ} \mathrm{C}$; Spectroscopic data were found to be similar to those reported by Kumar et al.

Ethyl (2-(5-(4-nitrobenzylidene)-2,4-dioxothiazolidin-3-yl)acetyl)glycinate (13aiii). A reaction of 4nitrobenzaldehyde $(0.58 \mathrm{~g}, 3.84 \mathrm{mmol})$ and $12 \mathrm{a}(1.04 \mathrm{~g}, 3.84 \mathrm{mmol})$ gave compound 13aiii as a white solid $(1.57 \mathrm{~g}, 98 \%) ; \mathrm{m} . \mathrm{p}=204-208{ }^{\circ} \mathrm{C} ; \mathrm{IR}\left(\mathrm{KBr} \mathrm{cm}^{-1}\right): 3291,3084,1744,1695,1661,1612,1154 ;{ }^{1} \mathrm{H} \mathrm{NMR}(400 \mathrm{MHz}$, DMSO-d $)) \delta_{H}(p p m) 8.80(\mathrm{~s}, 1 \mathrm{H}, \mathrm{NH}), 8.36(\mathrm{~d}, 2 \mathrm{H}, J 8.4 \mathrm{~Hz}, 2 \times \mathrm{Ar}-\mathrm{H}), 8.10(\mathrm{~s}, 1 \mathrm{H}$, Aryl-H), $7.93(\mathrm{~d}, 2 \mathrm{H}, J 8.4,2 \times$ $\operatorname{Ar}-\mathrm{H}$ ), $4.37\left(\mathrm{~s}, 2 \mathrm{H}, \mathrm{CH}_{2} \mathrm{~N}\right.$ ), $4.10\left(\mathrm{q}, 2 \mathrm{H}, J 7.2 \mathrm{~Hz}, \mathrm{OCH}_{2} \mathrm{CH}_{3}\right), 3.90$ (d, $\left.2 \mathrm{H}, J 5.2 \mathrm{~Hz}, \mathrm{CH}_{2} \mathrm{NH}\right), 1.20(\mathrm{t}, 3 \mathrm{H}, J 7.2 \mathrm{~Hz}$, $\left.\mathrm{CH}_{3} \mathrm{CH}_{2} \mathrm{O}\right) ;{ }^{13} \mathrm{C}$ NMR (100 MHz, DMSO-d $), \delta_{\mathrm{C}}(\mathrm{ppm}) 169.9(\mathrm{C}=\mathrm{O}), 167.0(\mathrm{C}=\mathrm{O}), 166.0(\mathrm{C}=\mathrm{O}), 165.4(\mathrm{C}=\mathrm{O}), 148.2$ $\left(\mathrm{Ar}-\mathrm{CNO}_{2}\right), 139.6(\mathrm{Ar}-\mathrm{C}), 131.6(2 \times \mathrm{Ar}-\mathrm{CH}), 131.3$ (Aryl-CH), $125.9(\mathrm{Ar}-\mathrm{C}), 124.8(2 \times \mathrm{Ar}-\mathrm{CH}), 61.0\left(\mathrm{OCH}_{2} \mathrm{CH}_{3}\right)$, $43.9\left(\mathrm{CH}_{2} \mathrm{~N}\right), 41.3\left(\mathrm{CH}_{2} \mathrm{NH}\right), 14.5\left(\mathrm{CH}_{3} \mathrm{CH}_{2} \mathrm{O}\right)$; HRMS (ESI-TOF) $\mathrm{m} / \mathrm{z}$; calculated for $\mathrm{C}_{16} \mathrm{H}_{15} \mathrm{~N}_{3} \mathrm{O}_{7} \mathrm{~S}+\mathrm{H}$ : 394.0631, found $\mathrm{M}^{+}+\mathrm{H}: 394.0634$.

Ethyl (2-(5-(4-hydroxy-3-methoxybenzylidene)-2,4-dioxothiazolidin-3-yl)acetyl)glycinate (13aiv).

A reaction of vanillin $(0.58 \mathrm{~g}, 3.84 \mathrm{mmol})$ and $12 \mathrm{a}(1.02 \mathrm{~g}, 3.84 \mathrm{mmol})$ gave compound 13aiv as a yellow solid $\left(1.47\right.$ g, 97\%); m.p = 233-234 ${ }^{\circ} \mathrm{C} ; \mathrm{IR}\left(\mathrm{KBr} \mathrm{cm}^{-1}\right)$ : 3378, 3294, 2987, 1742, 1727, 1677, 1657, 1168; ${ }^{1} \mathrm{H}$ NMR (400 $\left.\mathrm{MHz}, \mathrm{DMSO}-d_{6}\right) \delta_{\mathrm{H}}(\mathrm{ppm}) 8.78(\mathrm{~s}, 1 \mathrm{H}, \mathrm{NH}), 7.86(\mathrm{~s}, 1 \mathrm{H}, \operatorname{Aryl}-\mathrm{H}), 7.20(\mathrm{~s}, 1 \mathrm{H}, \mathrm{Ar}-\mathrm{H}), 7.12(\mathrm{~d}, 1 \mathrm{H}, J 8.0 \mathrm{~Hz}, \mathrm{Ar}-\mathrm{H})$, $6.92(\mathrm{~d}, 1 \mathrm{H}, J 8.1 \mathrm{~Hz}, \mathrm{Ar}-\mathrm{H}), 4.33\left(\mathrm{~s}, 2 \mathrm{H}, \mathrm{CH}_{2} \mathrm{~N}\right), 4.09\left(\mathrm{q}, 2 \mathrm{H}, J 6.8 \mathrm{~Hz}, \mathrm{CH}_{2} \mathrm{OCH}_{3}\right), 3.88\left(\mathrm{~d}, 2 \mathrm{H}, J 5.2 \mathrm{~Hz}, \mathrm{CH}_{2} \mathrm{~N}\right), 3.82$ $\left(\mathrm{s}, 3 \mathrm{H}, \mathrm{OCH}_{3}\right), 1.19$ (t, 3H, J 7.2 Hz, $\left.\mathrm{CH}_{3} \mathrm{CH}_{2} \mathrm{O}\right) ;{ }^{13} \mathrm{C}$ NMR (100 MHz, DMSO-d $)$ ), $\delta_{c}(\mathrm{ppm})$ 169.9(C=O), 167.7 (C=O), $166.3(\mathrm{C}=\mathrm{O}), 165.8(\mathrm{C}=\mathrm{O}), 151.1\left(\mathrm{Ar}-\mathrm{COCH}_{3}\right), 148.7$ (Ar-COH), 134.7 (Aryl-CH), $125.0(\mathrm{Ar}-\mathrm{CH}), 124.2(\mathrm{Ar}-\mathrm{C}), 116.9$ (Ar-CH), $116.6(\mathrm{Ar}-\mathrm{C}), 114,7(\mathrm{Ar}-\mathrm{CH}), 61.0\left(\mathrm{OCH}_{2} \mathrm{CH}_{3}\right), 56.1\left(\mathrm{OCH}_{3}\right), 43.5\left(\mathrm{CH}_{2} \mathrm{~N}\right), 41.3\left(\mathrm{CH}_{2} \mathrm{NH}\right), 14.5\left(\mathrm{CH}_{3} \mathrm{CH}_{2} \mathrm{O}\right)$; HRMS (ESI-TOF) $m / z$; calculated for $\mathrm{C}_{17} \mathrm{H}_{18} \mathrm{~N}_{2} \mathrm{O}_{7} \mathrm{~S}$ : 394.0835, found $\mathrm{M}^{+}: 394.0838$.

Ethyl (2-(5-(benzo[d][1,3]dioxol-5-ylmethylene)-2,4-dioxothiazolidin-3-yl)acetyl) glycinate (13av). A reaction of piperonal $(0.58 \mathrm{~g}, 3.84 \mathrm{mmol})$ and $12 \mathrm{a}(1.01 \mathrm{~g}, 3.84 \mathrm{mmol})$ gave compound 13av as a yellow solid (0.81 $\mathrm{g}$, $54 \%), \mathrm{m} . \mathrm{p}=211-212^{\circ} \mathrm{C}$; IR $\left(\mathrm{KBr} \mathrm{cm}^{-1}\right): 3287,3094,1730,1682,1658,1623,1155 ;{ }^{1} \mathrm{H}$ NMR (400 MHz, DMSO$\left.d_{6}\right) \delta_{\mathrm{H}}(\mathrm{ppm}) 8.82(\mathrm{t}, 1 \mathrm{H}, \mathrm{J} 5.7 \mathrm{~Hz}, \mathrm{NH}), 7.94(\mathrm{~s}, 1 \mathrm{H}, \mathrm{Aryl}-\mathrm{H}), 7.28-7.25(\mathrm{~m}, 2 \mathrm{H}, 2 \times \mathrm{Ar}-\mathrm{H}), 7.17(\mathrm{~d}, 1 \mathrm{H}, J 8.0 \mathrm{~Hz}, \mathrm{Ar}-$ $\mathrm{H}), 6.20\left(\mathrm{~s}, 1 \mathrm{H}, \mathrm{OCH}_{2} \mathrm{O}\right), 4.39\left(\mathrm{~s}, 2 \mathrm{H}, \mathrm{CH}_{2} \mathrm{~N}\right), 4.15\left(\mathrm{q}, 2 \mathrm{H}, J 7.1 \mathrm{~Hz}, \mathrm{OCH}_{2} \mathrm{CH}_{3}\right), 3.93\left(\mathrm{~d}, 2 \mathrm{H}, J 5.8 \mathrm{~Hz}, \mathrm{CH}_{2} \mathrm{NH}\right), 1.25$ $\left(\mathrm{t}, 3 \mathrm{H}, J 7.1 \mathrm{~Hz}, \mathrm{CH}_{3} \mathrm{CH}_{2} \mathrm{O}\right) ;{ }^{13} \mathrm{C} \mathrm{NMR}\left(100 \mathrm{MHz}, \mathrm{DMSO}-d_{6}\right), \delta \mathrm{c}(\mathrm{ppm}) 169.9(\mathrm{C}=0), 167.5(\mathrm{C}=0), 166.2(\mathrm{C}=0)$, 165.7 (C=O), 150.1 (Ar-C), 148.3 (Ar-C), 133.9 (Aryl-CH), 127.5 (Ar-C), 126.6 (Ar-CH), 119.0 (Ar-C), 109.8 (Ar$\mathrm{CH}), 109.7(\mathrm{Ar}-\mathrm{CH}), 102.6\left(\mathrm{OCH}_{2} \mathrm{O}\right), 61.0\left(\mathrm{OCH}_{2} \mathrm{CH}_{3}\right), 43.6\left(\mathrm{CH}_{2} \mathrm{~N}\right), 41.3\left(\mathrm{CH}_{2} \mathrm{NH}\right), 14.5\left(\mathrm{CH}_{3} \mathrm{CH}_{2} \mathrm{O}\right)$; HRMS (ESITOF) $\mathrm{m} / \mathrm{z}$; calculated for $\mathrm{C}_{17} \mathrm{H}_{16} \mathrm{~N}_{2} \mathrm{O}_{7} \mathrm{~S}: 392.0678$, found $\mathrm{M}^{+}: 392.0681$. 
Ethyl (2-(5-(4-methylbenzylidene)-2,4-dioxothiazolidin-3-yl)acetyl)glycinate (13avi). A reaction of $p$ tolualdehyde $(0.30 \mathrm{ml}, 2.28 \mathrm{mmol})$ and $12 \mathrm{a}(0.82 \mathrm{~g}, 2.28 \mathrm{mmol})$ gave compound 13avi as a white solid (0.38 g, $46 \%) ; \mathrm{m} . \mathrm{p}=220-22{ }^{\circ} \mathrm{C}$; IR $\left(\mathrm{KBr} \mathrm{cm}^{-1}\right): 3279,3045,1740,1663,1658,1612,1150 ;{ }^{1} \mathrm{H} \mathrm{NMR}\left(400 \mathrm{MHz}, \mathrm{DMSO}-d_{6}\right)$ $\delta_{\mathrm{H}}(\mathrm{ppm}) 8.77(\mathrm{t}, 1 \mathrm{H}, J 5.6 \mathrm{~Hz}, \mathrm{NH}), 7.93(\mathrm{~s}, 1 \mathrm{H}, \mathrm{Aryl}-\mathrm{H}), 7.54(\mathrm{~d}, 2 \mathrm{H}, J 8.0 \mathrm{~Hz}, 2 \times \mathrm{Ar}-\mathrm{H}), 7.37$ (d, $2 \mathrm{H}, J 8.0 \mathrm{~Hz}, 2 \times$ $\mathrm{Ar}-\mathrm{H}$ ), 4.34 (s, 2H, CH $\mathrm{CH}_{2} \mathrm{~N}$ ), 4.09 (q, $2 \mathrm{H}, J 7.2 \mathrm{~Hz}, \mathrm{OCH}_{2} \mathrm{CH}_{3}$ ), 3.89 (d, $\left.2 \mathrm{H}, J 6.0 \mathrm{~Hz}, \mathrm{CH}_{2} \mathrm{NH},\right), 2.37\left(\mathrm{~s}, 3 \mathrm{H}, \mathrm{CH}_{3}\right), 1.19$ (t, 3H, J $\left.7.2 \mathrm{~Hz}, \mathrm{CH}_{3} \mathrm{CH}_{2} \mathrm{O}\right) ;{ }^{13} \mathrm{C}$ NMR (100 MHz, DMSO-d $)$, $\delta_{c}(\mathrm{ppm}) 169.9$ (C=0), 167.5 (C=O), 166.7 (C=O),

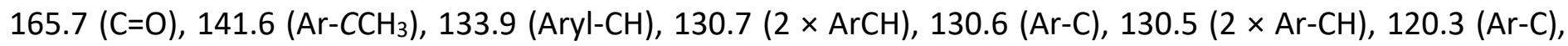
$61.0\left(\mathrm{OCH}_{2} \mathrm{CH}_{3}\right), 43.7\left(\mathrm{CH}_{2} \mathrm{~N}\right), 41.3\left(\mathrm{CH}_{2} \mathrm{NH}\right), 21.6\left(\mathrm{ArCH}_{3}\right), 14.6\left(\mathrm{CH}_{3} \mathrm{CH}_{2} \mathrm{O}\right) ; \mathrm{HRMS}$ (ESI-TOF) $\mathrm{m} / \mathrm{z}$; calculated for $\mathrm{C}_{17} \mathrm{H}_{18} \mathrm{~N}_{2} \mathrm{O}_{5} \mathrm{~S}+\mathrm{H}: 363.0936$ found $\mathrm{M}^{+}+\mathrm{H}: 363.0939$.

Ethyl (2-(5-(4-fluorobenzylidene)-2,4-dioxothiazolidin-3-yl)acetyl)glycinate (13avii). A reaction of 4fluorobenzaldehyde $(0.31 \mathrm{ml}, 2.88 \mathrm{mmol})$ and $12 \mathrm{a}(075 \mathrm{~g}, 2.88 \mathrm{mmol})$ gave compound 13avii as a yellow solid $(0.12 \mathrm{~g}, 12 \%) ; \mathrm{m} . \mathrm{p}=169-170{ }^{\circ} \mathrm{C} ; \mathrm{IR}\left(\mathrm{KBr} \mathrm{cm}^{-1}\right): 3299,2922,1740,1683,1658,1612,1180 ;{ }^{1} \mathrm{H} \mathrm{NMR}(400 \mathrm{MHz}$, DMSO-d $\left.d_{6}\right) \delta_{H}(\mathrm{ppm}) 8.83(\mathrm{t}, 1 \mathrm{H}, J 6.0 \mathrm{~Hz}, \mathrm{NH}), 8.05(\mathrm{~s}, 1 \mathrm{H}, \mathrm{Aryl}-\mathrm{H}), 7.81$ (dd, $\left.2 \mathrm{H},{ }^{4} J_{F H} 8.8 \mathrm{~Hz}, J_{H H} 5.6 \mathrm{~Hz}, 2 \times \mathrm{Ar}-\mathrm{H}\right)$, $7.48\left(\mathrm{dd}, 2 \mathrm{H}, 3^{3} \mathrm{JH}_{F H} 17.6 \mathrm{~Hz}, J_{H H} 8.8 \mathrm{~Hz}, 2 \times \mathrm{Ar}-\mathrm{H}\right), 4.40\left(\mathrm{~s}, 2 \mathrm{H}, \mathrm{CH}_{2} \mathrm{~N}\right), 4.21\left(\mathrm{q}, 2 \mathrm{H}, J 6.0 \mathrm{~Hz}, \mathrm{CH}_{2} \mathrm{OCH}_{3}\right), 3.95(\mathrm{~d}, 2 \mathrm{H}, J$ $5.6 \mathrm{~Hz}, \mathrm{CH}_{2} \mathrm{NH}$ ) $), 1.27$ (t, 3H, J $\left.8.4 \mathrm{~Hz}, \mathrm{CH}_{3} \mathrm{CH}_{2} \mathrm{O}\right) ;{ }^{19} \mathrm{~F}$ NMR (376.5 MHz, DMSO-d 6 ) $\delta_{\mathrm{F}}(\mathrm{ppm})$-107.92-(-109.06) (m,

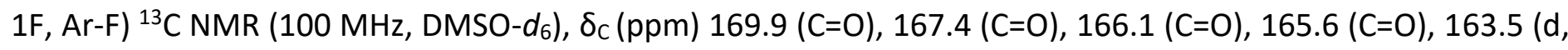
1J $250.0 \mathrm{~Hz}, \mathrm{Ar}-\mathrm{CF}$ ), 133.2 (d, 3 J $9.0 \mathrm{~Hz}, 2 \times \mathrm{Ar}-\mathrm{CH}$ ), 132.8 (Aryl-CH), 130.0 (d, ${ }^{4}$ J $\left.3.0 \mathrm{~Hz}, \mathrm{Ar}-\mathrm{C}\right), 121.3$ (Ar-C), 117.1 (d, $\left.{ }^{2} \mathrm{~J} 22.0 \mathrm{~Hz}, 2 \times \mathrm{Ar}-\mathrm{CH}\right), 61.1\left(\mathrm{OCH}_{2} \mathrm{CH}_{3}\right), 43.7\left(\mathrm{CH}_{2} \mathrm{~N}\right), 41.3\left(\mathrm{CH}_{2} \mathrm{NH}\right), 14.5\left(\mathrm{CH}_{3} \mathrm{CH} \mathrm{H}_{2} \mathrm{O}\right)$; HRMS (ESI-TOF) $\mathrm{m} / \mathrm{z}$; calculated for $\mathrm{C}_{16} \mathrm{H}_{15} \mathrm{FN}_{2} \mathrm{O}_{5} \mathrm{~S}+\mathrm{H}: 367.0686$ found $\mathrm{M}^{+}+\mathrm{H}: 367.0689$.

Ethyl (2-(5-benzylidene-2,4-dioxothiazolidin-3-yl)acetyl)alaninate (13bi). A reaction of benzaldehyde (0.40 $\mathrm{ml}, 3.65 \mathrm{mmol}$ ) and $\mathbf{1 2 b}$ (1.05 g, $3.65 \mathrm{mmol}$ ) gave compound 13bi as a white solid (0.64 g, 50\%); m.p = 167-168 ${ }^{\circ} \mathrm{C}$; IR $\left(\mathrm{KBr} \mathrm{cm}^{-1}\right): 3287,3087,1734,1687,1660,1608,1149 ;{ }^{1} \mathrm{H}$ NMR $\left(400 \mathrm{MHz}, \mathrm{DMSO}-d_{6}\right) \delta_{\mathrm{H}}(\mathrm{ppm}) 8.79(\mathrm{~d}$, $1 \mathrm{H}$, J $6.8 \mathrm{~Hz}, \mathrm{NH}), 7.96(\mathrm{~s}, 1 \mathrm{H}, \mathrm{Aryl}-\mathrm{H}), 7.65(\mathrm{~d}, 2 \mathrm{H}, J 7.2 \mathrm{~Hz}, 2 \times \mathrm{Ar}-\mathrm{H}), 7.58-7.5(\mathrm{~m}, 3 \mathrm{H}, 3 \times \mathrm{Ar}-\mathrm{H}), 4.29(\mathrm{~s}, 2 \mathrm{H}$, $\mathrm{CH}_{2} \mathrm{~N}$ ), 4.26 (quint, $2 \mathrm{H}, J 7.2 \mathrm{~Hz}, \mathrm{CHNH}$ ), 4.08 (q, 2H, J $\left.6.2 \mathrm{~Hz}, \mathrm{OCH}_{2} \mathrm{CH}_{3}\right), 1.30\left(\mathrm{~d}, 3 \mathrm{H}, J 7.2 \mathrm{~Hz}, \mathrm{CH}_{3} \mathrm{CH}\right) 1.18(\mathrm{t}$, $3 \mathrm{H}$, J 7.2 Hz, $\left.\mathrm{CH}_{3} \mathrm{CH}_{2} \mathrm{O}\right) ;{ }^{13} \mathrm{C} \mathrm{NMR}(100 \mathrm{MHz}$, DMSO-d $), \delta_{\mathrm{c}}(\mathrm{ppm}) 172.7$ (C=O), 167.5 (C=O), 165.7 (C=O), 165.5

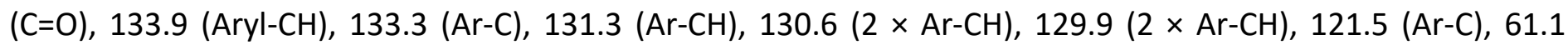
$\left(\mathrm{OCH}_{2} \mathrm{CH}_{3}\right), 48.4\left(\mathrm{CH}_{2} \mathrm{NH}\right), 43.6\left(\mathrm{CH}_{2} \mathrm{~N}\right), 17.4\left(\mathrm{CH}_{3} \mathrm{CH}\right), 14.4\left(\mathrm{CH}_{3} \mathrm{CH}_{2} \mathrm{O}\right)$; HRMS (ESI-TOF) $\mathrm{m} / \mathrm{z}$; calculated for $\mathrm{C}_{17} \mathrm{H}_{18} \mathrm{~N}_{2} \mathrm{O}_{5} \mathrm{~S}: 362.0936$, found $\mathrm{M}^{+}: 362.0939$.

Ethyl (2-(5-(4-methoxybenzylidene)-2,4-dioxothiazolidin-3-yl)acetyl)alaninate (13bii). A reaction of $p$ anisaldehyde $(0.45 \mathrm{ml}, 3.65 \mathrm{mmol})$ and $12 \mathrm{~b}(1.02 \mathrm{~g}, 3.65 \mathrm{mmol})$ gave compound 13bii as a brown solid (1.34 g, $98 \%) ; \mathrm{m} . \mathrm{p}=208-209{ }^{\circ} \mathrm{C} ; \mathrm{IR}\left(\mathrm{KBr} \mathrm{cm}^{-1}\right): 3296,3085,1733,1681,1659,1183 ;{ }^{1} \mathrm{H} \mathrm{NMR}\left(400 \mathrm{MHz}, \mathrm{DMSO}-d_{6}\right) \delta_{\mathrm{H}}$ (ppm) 8.77 (d, 1H, J $6.6 \mathrm{~Hz}, \mathrm{NH}), 7.92$ (s, 1H, Aryl-H), 7.62 (d, 2H, J 8.3 Hz, $2 \times \mathrm{Ar}-\mathrm{H}), 7.12(\mathrm{~d}, 2 \mathrm{H}, J 8.3 \mathrm{~Hz}, 2 \times \mathrm{Ar}-$ $\mathrm{H}), 4.32\left(\mathrm{~s}, 2 \mathrm{H}, \mathrm{CH}_{2} \mathrm{~N}\right.$ ), 4.25 (quint, $1 \mathrm{H}, J 6.8 \mathrm{~Hz}, \mathrm{CHNH}$ ), 4.08 (q, $\left.2 \mathrm{H}, J 7.8 \mathrm{~Hz}, \mathrm{OCH}_{2} \mathrm{CH}_{3}\right), 3.84(\mathrm{~s}, 3 \mathrm{H}, \mathrm{OCH}$ ), 1.30 (d, $\left.3 \mathrm{H}, J 7.0 \mathrm{~Hz}, \mathrm{CH}_{3} \mathrm{CH}\right) 1.19\left(\mathrm{t}, 3 \mathrm{H}, J 6.9 \mathrm{~Hz}, \mathrm{CH}_{3} \mathrm{CH}_{2} \mathrm{O}\right) ;{ }^{13} \mathrm{C}$ NMR (100 MHz, DMSO-d $\left.)_{6}\right), \delta_{c}(p p m) 172.6(\mathrm{C}=0)$, $167.5(\mathrm{C}=\mathrm{O}), 165.8(\mathrm{C}=\mathrm{O}), 165.5(\mathrm{C}=\mathrm{O}), 161.7\left(\mathrm{Ar}-\mathrm{COCH}_{3}\right), 133.8(\mathrm{Aryl}-\mathrm{CH}), 132.8(2 \times \mathrm{Ar}-\mathrm{CH}), 125.8(\mathrm{Ar}-\mathrm{C})$, 118.2 (Ar-C), $115.5(2 \times \mathrm{Ar}-\mathrm{CH}), 61.0\left(\mathrm{OCH}_{2} \mathrm{CH}_{3}\right), 56.0\left(\mathrm{OCH}_{3}\right), 48.4(\mathrm{CHNH}), 43.5\left(\mathrm{CH}_{2} \mathrm{~N}\right), 17.5\left(\mathrm{CH}_{3} \mathrm{CH}\right), 14.5$ $\left(\mathrm{CH}_{3} \mathrm{CH}_{2} \mathrm{O}\right)$; HRMS (ESI-TOF) $\mathrm{m} / \mathrm{z}$; calculated for $\mathrm{C}_{18} \mathrm{H}_{20} \mathrm{~N}_{2} \mathrm{O}_{6} \mathrm{~S}: 392.4260$, found $\mathrm{M}^{+}: 392.4262$.

Ethyl (2-(5-(4-nitrobenzylidene)-2,4-dioxothiazolidin-3-yl)acetyl)alaninate (13biii). A reaction of 4nitrobenzaldehyde $(0.55 \mathrm{~g}, 3.65 \mathrm{mmol})$ and $12 \mathrm{~b}(1.02 \mathrm{~g}, 3.65 \mathrm{mmol})$ in ethanol $(20 \mathrm{ml})$ gave compound $13 \mathrm{biii}$ as a brown solid $(1.31 \mathrm{~g}, 90 \%) ; \mathrm{m} . \mathrm{p}=223-224{ }^{\circ} \mathrm{C}$; IR $\left(\mathrm{KBr} \mathrm{cm}^{-1}\right): 3325,3035,1760,1737,1687,1671,1159 ;{ }^{1} \mathrm{H}$ NMR $\left(400 \mathrm{MHz}, \mathrm{DMSO}-d_{6}\right) \delta_{\mathrm{H}}(\mathrm{ppm}) 8.88(\mathrm{~d}, 1 \mathrm{H}, J 6.9 \mathrm{~Hz}, \mathrm{NH}), 8.43(\mathrm{~d}, 2 \mathrm{H}, J 8.8 \mathrm{~Hz}, \mathrm{Ar}-\mathrm{H}), 8.16(\mathrm{~s}, 1 \mathrm{H}, \mathrm{Aryl}-\mathrm{H})$, $7.99(\mathrm{~d}, 2 \mathrm{H}, J 8.4 \mathrm{~Hz}, 2 \times \mathrm{Ar}-\mathrm{H}), 4.42\left(\mathrm{~s}, 2 \mathrm{H}, \mathrm{CH}_{2} \mathrm{~N}\right.$ ), 4.34 (quint, $\left.1 \mathrm{H}, J 7.2 \mathrm{~Hz}, \mathrm{CHNH}\right), 4.16(\mathrm{q}, 2 \mathrm{H}, J 6.0 \mathrm{~Hz}$, $\left.\mathrm{OCH}_{2} \mathrm{CH}_{3}\right), 1.38\left(\mathrm{~d}, 3 \mathrm{H}, J 7.3 \mathrm{~Hz}, \mathrm{CH}_{3} \mathrm{CH}\right), 1.29\left(\mathrm{t}, 3 \mathrm{H}, J 7.1 \mathrm{~Hz}, \mathrm{CH}_{3} \mathrm{CH}_{2} \mathrm{O}\right) ;{ }^{13} \mathrm{C} \mathrm{NMR}\left(100 \mathrm{MHz}, \mathrm{DMSO}-d_{6}\right), \delta_{c}$ (ppm) $172.6(\mathrm{C}=\mathrm{O}), 167.0(\mathrm{C}=\mathrm{O}), 165.4(\mathrm{C}=\mathrm{O}), 165.3(\mathrm{C}=\mathrm{O}), 148.1\left(\mathrm{Ar}-\mathrm{CNO}_{2}\right), 139.6(\mathrm{Ar}-\mathrm{C}), 131.56(2 \times \mathrm{Ar}-\mathrm{CH})$, 
131.3 (Aryl-CH), 126.4 (Ar-C), $125.8(2 \times \mathrm{Ar}-\mathrm{CH}), 61.1\left(\mathrm{OCH}_{2} \mathrm{CH}_{3}\right), 48.4\left(\mathrm{CH}_{2} \mathrm{NH}\right), 43.8\left(\mathrm{CH}_{2} \mathrm{~N}\right), 17.5\left(\mathrm{CH}{ }_{3} \mathrm{CH}\right)$, $14.44\left(\mathrm{CH}_{3} \mathrm{CH}_{2} \mathrm{O}\right)$; HRMS (ESI-TOF) $\mathrm{m} / 2$; calculated for $\mathrm{C}_{17} \mathrm{H}_{17} \mathrm{~N}_{3} \mathrm{O} \mathrm{O}_{7} \mathrm{~S}: 407,0787$, found $\mathrm{M}^{+}: 407.0786$.

Ethyl (2-(5-(4-hydroxy-3-methoxybenzylidene)-2,4-dioxothiazolidin-3-yl)acetyl)alaninate (13biv). A reaction of vanillin $(0.55 \mathrm{~g}, 3.65 \mathrm{mmol})$ and $12 \mathrm{~b}(1.03 \mathrm{~g}, 3.65 \mathrm{mmol})$ gave compound 13biv as a yellow solid (0.88 g, $61 \%) ; \mathrm{m} . \mathrm{p}=183-184{ }^{\circ} \mathrm{C}$; IR ( $\left.\mathrm{KBr} \mathrm{cm}^{-1}\right): 3294,2954,1737,1720,1682,1654,1168 ;{ }^{1} \mathrm{H} \mathrm{NMR}\left(400 \mathrm{MHz}, \mathrm{DMSO}-d_{6}\right)$ $\delta_{\mathrm{H}}(\mathrm{ppm}) 8.76(\mathrm{~d}, 1 \mathrm{H}, J 6.9 \mathrm{~Hz}, \mathrm{NH}), 7.87$ (s, 1H, Aryl-H), 7.22 (d, 1H, J $1.6 \mathrm{~Hz} \mathrm{Ar}-\mathrm{CH}$ ), 7.12 (dd, $1 \mathrm{H}, J_{1} 8.4 \mathrm{~Hz}, J_{2}$ 1.6 Hz Ar-CH), $6.94\left(\mathrm{~d}, 1 \mathrm{H}, J 8.0 \mathrm{~Hz}, \mathrm{Ar}-\mathrm{CH}\right.$ ), $4.25\left(\mathrm{~s}, 2 \mathrm{H}, \mathrm{CH}_{2} \mathrm{~N}\right.$ ), 4.24 (quint, $\left.1 \mathrm{H}, J 7.2 \mathrm{~Hz}, \mathrm{CHNH}\right), 4.07$ (q, 2H, J $\left.6.0 \mathrm{~Hz}, \mathrm{OCH}_{2} \mathrm{CH}_{3}\right), 3.83\left(\mathrm{~s}, 3 \mathrm{H}, \mathrm{OCH}_{3}\right), 1.30\left(\mathrm{~d}, 3 \mathrm{H}, J 7.6 \mathrm{~Hz}, \mathrm{CH}_{3} \mathrm{CH}\right) 1.18\left(\mathrm{t}, 3 \mathrm{H}, J 7.2 \mathrm{~Hz}, \mathrm{CH}_{3} \mathrm{CH}_{2} \mathrm{O}\right) ;{ }^{13} \mathrm{C} \mathrm{NMR}(100$ $\left.\mathrm{MHz}, \mathrm{DMSO}-d_{6}\right), \delta_{\mathrm{C}}(\mathrm{ppm}) 172.7(\mathrm{C}=\mathrm{O}), 167.6(\mathrm{C}=\mathrm{O}), 165.8(\mathrm{C}=\mathrm{O}), 165.6(\mathrm{C}=\mathrm{O}), 150.3\left(\mathrm{Ar}-\mathrm{COCH}_{3}\right), 148.5(\mathrm{Ar}-$ $\mathrm{COH}), 134.6$ (Aryl-CH), 124.8 (Ar-C), 124.7 (Ar-CH), 117.1 (Ar-C), 116.7 (Ar-CH), 114.7 (Ar-CH), $61.2\left(\mathrm{OCH}_{2} \mathrm{CH}_{3}\right)$, $56.1\left(\mathrm{OCH}_{3}\right), 48.4(\mathrm{CHN}), 43.5\left(\mathrm{CH}_{2} \mathrm{~N}\right), 17.5\left(\mathrm{CH}_{3} \mathrm{CH}\right), 14.4\left(\mathrm{CH}_{3} \mathrm{CH}_{2} \mathrm{O}\right)$; HRMS (ESI-TOF) $\mathrm{m} / z$; calculated for $\mathrm{C}_{18} \mathrm{H}_{20} \mathrm{~N}_{2} \mathrm{O}_{7} \mathrm{~S}: 408.0991$, found $\mathrm{M}^{+}: 408.0993$.

Ethyl (2-(5-(benzo[d] [1,3]dioxol-5-ylmethylene)-2,4-dioxothiazolidin-3-yl)acetyl)alaninate (13bv). A reaction of piperonal $(0.55 \mathrm{~g}, 3.65 \mathrm{mmol})$ and $\mathbf{1 2 b}(1.01 \mathrm{~g}, 3.65 \mathrm{mmol})$ gave compound $\mathbf{1 3 b v}$ as a yellow solid $(0.79 \mathrm{~g}$, 53\%), m.p $=174-175{ }^{\circ} \mathrm{C},{ }^{1} \mathrm{H}$ NMR $\left(400 \mathrm{MHz}, \mathrm{DMSO}-d_{6}\right) \delta_{\mathrm{H}}(\mathrm{ppm}) 8.77(\mathrm{~d}, 1 \mathrm{H}, J 7.0 \mathrm{~Hz}, \mathrm{NH}), 7.88(\mathrm{~s}, 1 \mathrm{H}, \mathrm{Aryl}-\mathrm{CH})$, 7.23-7.19 (m, $2 \mathrm{H}, 2 \times \mathrm{Ar}-\mathrm{H}), 7.11(\mathrm{~d}, 1 \mathrm{H}, J 8.0 \mathrm{~Hz}, \mathrm{Ar}-\mathrm{H}), 6.15\left(\mathrm{~s}, 2 \mathrm{H}, \mathrm{OCH}_{2} \mathrm{O}\right), 4.31\left(\mathrm{~s}, 2 \mathrm{H}, \mathrm{CH}_{2} \mathrm{~N}\right), 4.25$ (quint, $1 \mathrm{H}$, J 7.2, $\mathrm{CHNH}$ ), 4.08 (q, $\left.2 \mathrm{H}, J 6.4 \mathrm{~Hz}, \mathrm{OCH}_{2} \mathrm{CH}_{3}\right), 1.29$ (d, 3H, J 7.2, $\left.\mathrm{CH}_{3} \mathrm{CH}\right) 1.18\left(\mathrm{t}, 3 \mathrm{H}, J 6.8 \mathrm{~Hz}, \mathrm{CH}_{3} \mathrm{CH}_{2} \mathrm{O}\right),{ }^{13} \mathrm{C} \mathrm{NMR}$ (100 MHz, DMSO- $\left.d_{6}\right), \delta_{c}(p p m) 172.6(C=O), 167.4(C=0), 165.7(C=O), 165.5(C=0), 150.1(\operatorname{Ar}-C), 148.7(\operatorname{Ar}-\mathrm{C})$, 133.9 (Aryl-H), 127.5 (Ar-C), 126.7 (Ar-CH), 118.9 (Ar-C), $109.8(\mathrm{Ar}-\mathrm{CH}), 109.7$ (Ar-CH), $102.6\left(\mathrm{OCH}_{2} \mathrm{O}\right), 61.0$ $\left.\left(\mathrm{OCH}_{2} \mathrm{CH}_{3}\right), 48.4(\mathrm{CHNH}), 43.5\left(\mathrm{CH}_{2} \mathrm{~N}\right), 17.4\left(\mathrm{CH}_{3} \mathrm{CH}\right), 14.4\left(\mathrm{CH}_{3} \mathrm{CH}_{2} \mathrm{O}\right), \mathrm{IR}(\mathrm{KBr} \mathrm{cm})^{-1}\right): 3315,2998,1736,1680$, 1654, 1610 (C=O stretch), 1156, HRMS (ESI-TOF) $\mathrm{m} / \mathrm{z}$; calculated for $\mathrm{C}_{18} \mathrm{H}_{18} \mathrm{~N}_{2} \mathrm{O}{ }_{7} \mathrm{~S}$ : 406.0835, found $\mathrm{M}^{+}$: 406.0832.

Ethyl (2-(5-(4-methylbenzylidene)-2,4-dioxothiazolidin-3-yl)acetyl)alaninate (13bvi). A reaction of $p$ tolualdehyde $(0.32 \mathrm{ml}, 2.75 \mathrm{mmol})$ and $\mathbf{1 2 b}(0.82 \mathrm{~g}, 2.75 \mathrm{mmol})$ gave compound $13 \mathrm{bvi}$ as a white solid $(0.32 \mathrm{~g}$, $31 \%) ; \mathrm{m} . \mathrm{p}=213-214^{\circ} \mathrm{C}$; IR $\left(\mathrm{KBr} \mathrm{cm}^{-1}\right): 3282,2922,1735,1682,1659,1614,1148 ;{ }^{1} \mathrm{H}$ NMR (400 MHz, DMSO$\left.d_{6}\right) \delta_{\mathrm{H}}(\mathrm{ppm}) 8.77(\mathrm{~d}, 1 \mathrm{H}, J 7.2 \mathrm{~Hz}, \mathrm{NH}), 7.93(\mathrm{~s}, 1 \mathrm{H}, \operatorname{Aryl}-\mathrm{H}), 7.54(\mathrm{~d}, 2 \mathrm{H}, J 8.0 \mathrm{~Hz}, 2 \times \mathrm{Ar}-\mathrm{H}), 7.37(\mathrm{~d}, 2 \mathrm{H}, J 8.0 \mathrm{~Hz}$, $2 \times \mathrm{Ar}-\mathrm{H}$ ), $4.28\left(\mathrm{~s}, 2 \mathrm{H}, \mathrm{CH}_{2} \mathrm{~N}\right.$ ), 4.25 (quint, $1 \mathrm{H}, \mathrm{J} 7.2 \mathrm{~Hz}, \mathrm{CHNH}$ ), 4.09 (q, $\left.2 \mathrm{H}, J 7.4 \mathrm{~Hz}, \mathrm{OCH}_{2} \mathrm{CH}_{3}\right), 2.51\left(\mathrm{~s}, 3 \mathrm{H}, \mathrm{CH}_{3}\right)$, $1.30\left(\mathrm{~d}, 3 \mathrm{H}, J 7.2 \mathrm{~Hz}, \mathrm{CH}_{3} \mathrm{CH}\right) 1.14\left(\mathrm{t}, 3 \mathrm{H}, J 6.8 \mathrm{~Hz}, \mathrm{CH}_{3} \mathrm{CH}_{2} \mathrm{O}\right) ;{ }^{13} \mathrm{C} \mathrm{NMR}\left(100 \mathrm{MHz}, \mathrm{DMSO}-d_{6}\right), \delta_{\mathrm{C}}(\mathrm{ppm}) 172.6$ $(\mathrm{C}=\mathrm{O}), 167.5(\mathrm{C}=\mathrm{O}), 165.7(\mathrm{C}=\mathrm{O}), 165.5(\mathrm{C}=\mathrm{O}), 141.6\left(\mathrm{Ar}-\mathrm{CCH}_{3}\right), 133.9(\mathrm{Aryl}-\mathrm{CH}), 130.7$ (2 $\times$ Ar-CH), $130.9(\mathrm{Ar}-\mathrm{C})$, $130.50(2 \times \mathrm{Ar}-\mathrm{CH}), 120.3(\mathrm{Ar}-\mathrm{C}), 61.0\left(\mathrm{OCH}_{2} \mathrm{CH}_{3}\right), 48.4(\mathrm{CHNH}), 43.6\left(\mathrm{CH}_{2} \mathrm{~N}\right), 21.6\left(\mathrm{Ar}-\mathrm{CH}_{3}\right) 17.5\left(\mathrm{CH}_{3} \mathrm{CH}\right), 14.4$ $\left(\mathrm{CH}_{3} \mathrm{CH}_{2} \mathrm{O}\right)$; HRMS (ESI-TOF) $\mathrm{m} / \mathrm{z}$; calculated for $\mathrm{C}_{18} \mathrm{H}_{20} \mathrm{~N}_{2} \mathrm{O}_{5} \mathrm{~S}+\mathrm{H}: 376.1093$, found $\mathrm{M}^{+}+\mathrm{H}: 377.1096$.

Ethyl (2-(5-(4-fluorobenzylidene)-2,4-dioxothiazolidin-3-yl)acetyl)alaninate (13bvii). A reaction of 4fluorobenzaldehyde $(0.30 \mathrm{ml}, 2.75 \mathrm{mmol})$ and $12 \mathrm{~b}(0.821 \mathrm{~g}, 2.75 \mathrm{mmol})$ gave compound $13 \mathrm{bvii}$ as a yellow solid $(0.11 \mathrm{~g}, 11 \%) ; \mathrm{m} . \mathrm{p}=203-204{ }^{\circ} \mathrm{C}$; IR $\left(\mathrm{KBr} \mathrm{cm}{ }^{-1}\right): 3285,2984,1733,1685,1657,16381183 ;{ }^{1} \mathrm{H}$ NMR $(400$ $\left.\mathrm{MHz}, \mathrm{DMSO}-d_{6}\right) \delta_{\mathrm{H}}(\mathrm{ppm}) 8.77(\mathrm{~d}, 1 \mathrm{H}, J 6.8 \mathrm{~Hz}, \mathrm{NH}), 7.99(\mathrm{~s}, 1 \mathrm{H}, \mathrm{Aryl}-\mathrm{H}), 7.73\left(\mathrm{dd}, 2 \mathrm{H},{ }^{4} J_{F H} 8.8 \mathrm{~Hz}, J_{H H} 5.6 \mathrm{~Hz}, 2 \times\right.$ $\operatorname{Ar}-\mathrm{H}$ ), $7.41\left(\mathrm{dd}, 2 \mathrm{H},{ }^{3} J_{F H} 17.6 \mathrm{~Hz}, J_{H H} 8.8 \mathrm{~Hz}, 2 \times \mathrm{Ar}-\mathrm{H}\right.$ ), $4.13\left(\mathrm{~s}, 2 \mathrm{H}, \mathrm{CH}_{2} \mathrm{~N}\right.$ ), 4.12 (quint, $1 \mathrm{H}, J 3.2 \mathrm{~Hz}, \mathrm{CHNH}$ ), 4.06 (q, $2 \mathrm{H}, J 2.8 \mathrm{~Hz}, \mathrm{OCH}_{2} \mathrm{CH}_{3}$ ), 1.42 (d, $\left.3 \mathrm{H}, J 7.2 \mathrm{~Hz}, \mathrm{CH}_{3} \mathrm{CH}\right) 1.23\left(\mathrm{t}, 3 \mathrm{H}, J 4.8 \mathrm{~Hz}, \mathrm{CH}_{3} \mathrm{CH}_{2} \mathrm{O}\right) ;{ }^{19} \mathrm{~F} \mathrm{NMR}(376.5 \mathrm{MHz}$, DMSO- $\left.d_{6}\right) \delta_{F}(p p m)-108.30-(-108.36)(\mathrm{m}, 1 \mathrm{~F}, \mathrm{Ar}-\mathrm{F}) ;{ }^{13} \mathrm{C}$ NMR (100 MHz, DMSO-d $\left.d_{6}\right), \delta_{\mathrm{C}}(\mathrm{ppm}) 172.6(\mathrm{C}=0), 167.3$ (C=O), 165.6 (C=O), 165.4 (C=O), 163.5 (d, ${ }^{1}$ J $255.0 \mathrm{~Hz}, \mathrm{Ar}-\mathrm{C}$ ), 133.1 (d, 3J 9.0 Hz, $2 \times$ Ar-CH), 132.8 (Aryl-CH), 130.0 (d, 4J $3.0 \mathrm{~Hz}, \mathrm{Ar}-\mathrm{C}$ ), 121.3 (Ar-C), 117.1 (d, 2J $9.0 \mathrm{~Hz}, 2 \times \mathrm{Ar}-\mathrm{CH}), 61.0\left(\mathrm{OCH}_{2} \mathrm{CH}_{3}\right), 48.4(\mathrm{CHNH}), 43.6$ $\left(\mathrm{CH}_{2} \mathrm{~N}\right), 17.5\left(\mathrm{CH}_{3} \mathrm{CH}\right), 14.4\left(\mathrm{CH}_{3} \mathrm{CH}_{2} \mathrm{O}\right)$; HRMS (ESI-TOF) $\mathrm{m} / z$; calculated for $\mathrm{C}_{17} \mathrm{H}_{17} \mathrm{FN}_{2} \mathrm{O}_{5} \mathrm{~S}+\mathrm{H}$ : 381.0842, found $\mathrm{M}^{+}+\mathrm{H}: 381.0845$.

Ethyl (2-(5-(furan-2-ylmethylene)-2,4-dioxothiazolidin-3-yl)acetyl)alaninate (13bviii). A reaction of furfural $(0.46 \mathrm{ml}, 4.82 \mathrm{mmol})$ and $12 \mathrm{~b}(1.02 \mathrm{~g}, 4.82 \mathrm{mmol})$ in ethanol $(20 \mathrm{ml})$ gave compound 13 bviii as a white solid 
$(0.18 \mathrm{~g}, 34 \%) ; \mathrm{m} . \mathrm{p}=172-173{ }^{\circ} \mathrm{C} ; \mathrm{IR}\left(\mathrm{KBr} \mathrm{cm}^{-1}\right): 3296,3085,1733,1681,1659,1183 ;{ }^{1} \mathrm{H} \mathrm{NMR}(400 \mathrm{MHz}$, DMSO$\left.d_{6}\right) \delta_{H}(p p m) 8.75(\mathrm{~d}, 1 \mathrm{H}, J 6.8 \mathrm{~Hz}, \mathrm{NH}), 8.11(\mathrm{~d}, 1 \mathrm{H}, J 1.6 \mathrm{~Hz}, \mathrm{Ar}-\mathrm{CH}), 7.80(\mathrm{~s}, 1 \mathrm{H}, \operatorname{Aryl}-\mathrm{H}), 7.17(\mathrm{~d}, 1 \mathrm{H}, J 3.6 \mathrm{~Hz}$, $\operatorname{Ar}-\mathrm{CH}$ ), $6.78\left(\mathrm{dd}, 1 \mathrm{H}, J_{1} 3.6 \mathrm{~Hz}, J_{2} 1.6 \mathrm{~Hz}, \mathrm{Ar}-\mathrm{CH}\right), 4.22\left(\mathrm{~s}, 2 \mathrm{H}, \mathrm{CH}_{2} \mathrm{~N}\right.$ ), 4.11 (quint, $1 \mathrm{H}, J 2.4 \mathrm{~Hz}, \mathrm{CHNH}$ ), 4.06 (q, $\left.2 \mathrm{H}, J 4.4 \mathrm{~Hz}, \mathrm{OCH}_{2} \mathrm{CH}_{3}\right), 1.29$ (d, 3H, J $\left.7.2 \mathrm{~Hz}, \mathrm{CH}_{3} \mathrm{CH}\right) 1.18\left(\mathrm{t}, 3 \mathrm{H}, J 7.2 \mathrm{~Hz}, \mathrm{CH}_{3} \mathrm{CH}_{2} \mathrm{O}\right.$ ); ${ }^{13} \mathrm{C} \mathrm{NMR}(100 \mathrm{MHz}$, DMSO$\left.d_{6}\right), \delta_{c}(\mathrm{ppm}) 172.6(\mathrm{C}=\mathrm{O}), 168.2(\mathrm{C}=\mathrm{O}), 165.5(\mathrm{C}=\mathrm{O}), 165.4(\mathrm{C}=\mathrm{O}), 149.6(\mathrm{C}), 148.4(\mathrm{CH}), 120.3(\operatorname{Aryl}-\mathrm{CH}), 120.0$ (C-H) $118.1(\mathrm{C}), 114.1(\mathrm{CH}), 61.0\left(\mathrm{OCH}_{2} \mathrm{CH}_{3}\right), 48.4(\mathrm{CHNH}), 43.46\left(\mathrm{CH}_{2} \mathrm{~N}\right), 17.46\left(\mathrm{CH}_{3} \mathrm{CH}\right), 14.44\left(\mathrm{CH}_{3} \mathrm{CH}_{2} \mathrm{O}\right)$; HRMS (ESI-TOF) $m / z$; calculated for $\mathrm{C}_{15} \mathrm{H}_{16} \mathrm{~N}_{2} \mathrm{O}_{6} \mathrm{~S}+\mathrm{H}: 353.0729$, found $\mathrm{M}^{+}+\mathrm{H}: 353.0731$.

Ethyl 2-(2-(5-benzylidene-2,4-dioxothiazolidin-3-yl)acetamido)butanoate (13ci). A reaction of benzaldehyde $(0.32 \mathrm{ml}, 3.12 \mathrm{mmol})$ and $12 \mathrm{c}(0.90 \mathrm{~g}, 3.12 \mathrm{mmol})$ gave compound $13 \mathrm{ci}$ as a white solid $(0.16 \mathrm{~g}, 10 \%) ; \mathrm{m} . \mathrm{p} \mathrm{=}$ $175-176{ }^{\circ} \mathrm{C}$; IR $\left(\mathrm{KBr} \mathrm{cm}^{-1}\right)$ : 3304, 2923, 1735, 1687, 1661, 1607, 1149; ${ }^{1} \mathrm{H}$ NMR $\left(400 \mathrm{MHz}, \mathrm{DMSO}-d_{6}\right) \delta_{\mathrm{H}}(\mathrm{ppm})$ $8.71(\mathrm{~d}, 1 \mathrm{H}, \mathrm{J} 7.6 \mathrm{~Hz}, \mathrm{NH}), 7.97(\mathrm{~s}, 1 \mathrm{H}$, Aryl-H), 7.65 (d, 2H, J $7.2 \mathrm{~Hz}, 2 \times \mathrm{Ar}-\mathrm{H}), 7.61-7.50(\mathrm{~m}, 3 \mathrm{H}, 3 \times \mathrm{Ar}-\mathrm{H}), 4.35$ $\left(\mathrm{s}, 2 \mathrm{H}, \mathrm{CH}_{2} \mathrm{~N}\right.$ ), 4.20 (quint, $1 \mathrm{H}, J 3.6 \mathrm{~Hz}, \mathrm{CHNH}$ ), 4.09 (q, $\left.2 \mathrm{H}, J 3.6 \mathrm{~Hz}, \mathrm{OCH}_{2} \mathrm{CH}_{3}\right), 1.76-1.71\left(\mathrm{~m}, 1 \mathrm{H}, \mathrm{CH}_{a} \mathrm{CH}_{3}\right)$ 1.68$1.62\left(\mathrm{~m}, 1 \mathrm{H}, \mathrm{CH}_{b} \mathrm{CH}_{3}\right), 1.23\left(\mathrm{t}, 3 \mathrm{H}, J 7.2 \mathrm{~Hz}, \mathrm{CH}_{3} \mathrm{CH}_{2} \mathrm{O}\right), 0.90\left(\mathrm{t}, 3 \mathrm{H}, J 7.2 \mathrm{~Hz}, \mathrm{CH}_{3} \mathrm{CH}_{2}\right) ;{ }^{13} \mathrm{C} \mathrm{NMR}(100 \mathrm{MHz}$, DMSO$\left.d_{6}\right), \delta_{c}(p p m) 172.1(\mathrm{C}=\mathrm{O}), 167.5(\mathrm{C}=\mathrm{O}), 165.7(\mathrm{C}=\mathrm{O}), 165.1(\mathrm{C}=\mathrm{O}), 133.8$ (Aryl-CH), $133.3(\operatorname{Ar}-\mathrm{C}), 131.2(\mathrm{Ar}-\mathrm{CH})$, $130.6(2 \times \mathrm{Ar}-\mathrm{CH}), 129.9(2 \times \mathrm{Ar}-\mathrm{CH}), 121.6(\mathrm{Ar}-\mathrm{C}), 62.0\left(\mathrm{OCH}_{2} \mathrm{CH}_{3}\right), 54.0(\mathrm{CHNH}), 43.6\left(\mathrm{CH}_{2} \mathrm{~N}\right), 24.9\left(\mathrm{CH}_{2} \mathrm{CH}_{3}\right)$, $14.5\left(\mathrm{CH}_{3} \mathrm{CH}_{2} \mathrm{O}\right), 10.6\left(\mathrm{CH}_{3} \mathrm{CH}_{2}\right)$; HRMS (ESI-TOF) $\mathrm{m} / z$; calculated for $\mathrm{C}_{18} \mathrm{H}_{20} \mathrm{~N}_{2} \mathrm{O}_{5} \mathrm{~S}+\mathrm{H}$ : 377.1093, found $\mathrm{M}^{+}+\mathrm{H}$ : 377.1096.

Ethyl 2-(2-(5-(4-methoxybenzylidene)2,4-dioxothiazolidin-3-yl)acetamido)butanoate (13cii). A reaction of $p$ anisaldehyde $(0.26 \mathrm{ml}, 2.08 \mathrm{mmol})$ and $12 \mathrm{c}(0.60 \mathrm{~g}, 2.08 \mathrm{mmol})$ gave compound $13 \mathrm{cii}$ as a white solid $(0.31 \mathrm{~g}$, 37\%); m.p = 207-208 ${ }^{\circ} \mathrm{C} ;{ }^{1} \mathrm{H}$ NMR (400 MHz, DMSO-d $\left.)\right) \delta_{\mathrm{H}}(\mathrm{ppm}) 8.70(\mathrm{~d}, 1 \mathrm{H}, J 7.6 \mathrm{~Hz}, \mathrm{NH}), 7.93$ (s, 1H, Aryl-H), $7.62(\mathrm{~d}, 2 \mathrm{H}, J 8.8 \mathrm{~Hz}, 2 \times \mathrm{Ar}-\mathrm{H}), 7.13(\mathrm{~d}, 2 \mathrm{H}, J 8.8 \mathrm{~Hz}, 2 \times \mathrm{Ar}-\mathrm{H}), 4.15\left(\mathrm{~s}, 2 \mathrm{H}, \mathrm{CH}_{2} \mathrm{~N}\right.$ ), 4.12 (quint, $1 \mathrm{H}, J 2.8 \mathrm{~Hz}$, $\mathrm{CHNH}$ ), 4.07 (q, 2H, J $\left.3.6 \mathrm{~Hz}, \mathrm{OCH}_{2} \mathrm{CH}_{3}\right), 3.84\left(\mathrm{~s}, 3 \mathrm{H}, \mathrm{OCH}_{3}\right), 1.78-1.73\left(\mathrm{~m}, 1 \mathrm{H}, \mathrm{CH}_{a} \mathrm{CH}_{3}\right) 1.70-1.61(\mathrm{~m}, 1 \mathrm{H}$, $\left.\mathrm{CH}_{b} \mathrm{CH}_{3}\right), 1.19\left(\mathrm{t}, 3 \mathrm{H}, J 7.2 \mathrm{~Hz}, \mathrm{CH}_{3} \mathrm{CH}_{2} \mathrm{O}\right), 0.90\left(\mathrm{t}, 3 \mathrm{H}, J 7.2 \mathrm{~Hz}, \mathrm{CH}_{3} \mathrm{CH}_{2}\right) ;{ }^{13} \mathrm{C} \mathrm{NMR}\left(100 \mathrm{MHz}, \mathrm{DMSO}-d_{6}\right), \delta_{\mathrm{c}}(\mathrm{ppm})$ $172.0(\mathrm{C}=\mathrm{O}), 167.5(\mathrm{C}=\mathrm{O}), 165.8(2 \times \mathrm{C}=\mathrm{O}), 161.7\left(\mathrm{Ar}-\mathrm{COCH}_{3}\right), 133.9(\mathrm{Aryl}-\mathrm{CH}), 133.8(2 \times \mathrm{Ar}-\mathrm{CH}), 125.8(\mathrm{Ar}-\mathrm{C})$, 118.2 (Ar-C), $115.5(2 \times \mathrm{Ar}-\mathrm{CH}), 61.0\left(\mathrm{OCH}_{2} \mathrm{CH}_{3}\right), 56,0\left(\mathrm{OCH}_{3}\right), 54.0(\mathrm{CHNH}), 43.6\left(\mathrm{CH}_{2} \mathrm{~N}\right), 24.9\left(\mathrm{CH}_{2} \mathrm{CH}_{3}\right), 14.5$ $\left(\mathrm{CH}_{3} \mathrm{CH}_{2} \mathrm{O}\right)$, $10.6\left(\mathrm{CH}_{3} \mathrm{CH}_{2}\right)$; IR $\left(\mathrm{KBr} \mathrm{cm}{ }^{-1}\right): 3297,2978,1734,1685,1665,1602,1183$; HRMS (ESI-TOF) $\mathrm{m} / \mathrm{z}$; calculated for $\mathrm{C}_{19} \mathrm{H}_{22} \mathrm{~N}_{2} \mathrm{O}_{6} \mathrm{~S}+\mathrm{H}: 407.1199$, found $\mathrm{M}^{+}+\mathrm{H}: 407.1272$.

Ethyl 2-(2-(5-(4-methylbenzylidene)2,4-dioxothiazolidin-3-yl)acetamido)butanoate (13ciii). A reaction of $p$ tolualdehyde $(0.40 \mathrm{ml}, 3.47 \mathrm{mmol})$ and $12 \mathrm{c}(0.80 \mathrm{~g}, 3.47 \mathrm{mmol})$ gave compound 13ciii as a white solid $(0.11 \mathrm{~g}$, $8 \%) ; \mathrm{m} . \mathrm{p}=206-207^{\circ} \mathrm{C}$; IR $\left(\mathrm{KBr} \mathrm{cm}^{-1}\right): 3284,2987,1749,1685,1667,1606,1149 ;{ }^{1} \mathrm{H}$ NMR $\left(400 \mathrm{MHz}, \mathrm{DMSO}-d_{6}\right)$ $\delta_{\mathrm{H}}(\mathrm{ppm}) 8.71(\mathrm{~d}, 1 \mathrm{H}, J 7.6 \mathrm{~Hz}, \mathrm{NH}), 7.93(\mathrm{~s}, 1 \mathrm{H}, \mathrm{Aryl}-\mathrm{H}), 7.55(\mathrm{~d}, 2 \mathrm{H}, J 8.0 \mathrm{~Hz}, 2 \times \mathrm{Ar}-\mathrm{H}), 7.13(\mathrm{~d}, 2 \mathrm{H}, J 8.0 \mathrm{~Hz}, 2 \times$ $\mathrm{Ar}-\mathrm{H}$ ), $4.16\left(\mathrm{~s}, 2 \mathrm{H}, \mathrm{CH}_{2} \mathrm{~N}\right.$ ), 4.14 (quint, $1 \mathrm{H}, J 3.6 \mathrm{~Hz}, \mathrm{CHNH}$ ), 4.08 (q, $2 \mathrm{H}, J 3.6 \mathrm{~Hz}, \mathrm{OCH}_{2} \mathrm{CH}_{3}$ ), 2.37 (s, $\left.3 \mathrm{H}, \mathrm{Ar}-\mathrm{CH}_{3}\right)$, 1.77-1.71 (m, $\left.1 \mathrm{H}, \mathrm{CH}_{a} \mathrm{CH}_{3}\right)$ 1.68-1.62 (m, $\left.1 \mathrm{H}, \mathrm{CH}_{b} \mathrm{CH}_{3}\right), 1.19\left(\mathrm{t}, 3 \mathrm{H}, J 7.2 \mathrm{~Hz}, \mathrm{CH}_{3} \mathrm{CH}_{2} \mathrm{O}\right), 0.90(\mathrm{t}, 3 \mathrm{H}, J 7.2 \mathrm{~Hz}$, $\left.\mathrm{CH}_{3} \mathrm{CH}_{2}\right) ;{ }^{13} \mathrm{C}$ NMR (100 MHz, DMSO-d $), \delta_{c}(\mathrm{ppm}) 172.0(\mathrm{C}=\mathrm{O}), 167.5(\mathrm{C}=0), 165.8(2 \times \mathrm{C}=0), 141.6\left(\mathrm{Ar}-\mathrm{CCH}_{3}\right)$,

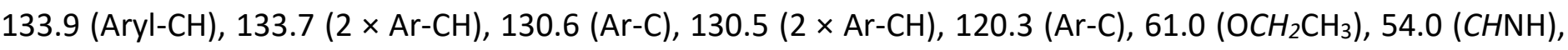
$43.6\left(\mathrm{CH}_{2} \mathrm{~N}\right), 24.9\left(\mathrm{CH}_{2} \mathrm{CH}_{3}\right), 21.4\left(\mathrm{Ar}-\mathrm{CH}_{3}\right), 14.5\left(\mathrm{CH}_{3} \mathrm{CH}_{2} \mathrm{O}\right), 10.6\left(\mathrm{CH}_{3} \mathrm{CH}_{2}\right)$; HRMS (ESI-TOF) $\mathrm{m} / z$; calculated for $\mathrm{C}_{19} \mathrm{H}_{22} \mathrm{~N}_{2} \mathrm{O}_{5} \mathrm{~S}+\mathrm{H}: 391.1249$, found $\mathrm{M}^{+}+\mathrm{H}: 391.1251$.

Ethyl 2-(2-(5-(4-hydroxybenzylidene)2,4-dioxothiazolidin-3-yl)acetamido)butanoate (13civ). A reaction of 4hydoxybenzaldehyde $(0.42 \mathrm{~g}, 2.77 \mathrm{mmol})$ and $12 \mathrm{c}(0.80 \mathrm{~g}, 2.77 \mathrm{mmol})$ gave compound 13civ as a white solid $(0.06 \mathrm{~g}, 6 \%) ; \mathrm{m} . \mathrm{p}=185-186^{\circ} \mathrm{C} ; \mathrm{IR}\left(\mathrm{KBr} \mathrm{cm}{ }^{-1}\right): 3287,3087,1734,1687,1660,1608,1149 ;{ }^{1} \mathrm{H} \mathrm{NMR}(400 \mathrm{MHz}$, DMSO-d $)_{6} \delta_{\mathrm{H}}(\mathrm{ppm}) 10.43(\mathrm{~s}, 1 \mathrm{H}, \mathrm{OH}), 8.70(\mathrm{~d}, 1 \mathrm{H}, J 7.6 \mathrm{~Hz}, \mathrm{NH}), 7.86(\mathrm{~s}, 1 \mathrm{H}$, Aryl-H), $7.51(\mathrm{~d}, 2 \mathrm{H}, J 8.8 \mathrm{~Hz}, 2 \times$ $\operatorname{Ar}-\mathrm{H}$ ), $6.93(\mathrm{~d}, 2 \mathrm{H}, J=8.8 \mathrm{~Hz}, 2 \times \mathrm{Ar}-\mathrm{H}), 4.33\left(\mathrm{~s}, 2 \mathrm{H}, \mathrm{CH}_{2} \mathrm{~N}\right.$ ), 4.17 (quint, $1 \mathrm{H}, J 2.8 \mathrm{~Hz}, \mathrm{CHNH}$ ), $4.09(\mathrm{q}, 2 \mathrm{H}, J 7.2$ $\left.\mathrm{Hz}, \mathrm{OCH}_{2} \mathrm{CH}_{3}\right), 1.77-1.71\left(\mathrm{~m}, 1 \mathrm{H}, \mathrm{CH}_{a} \mathrm{CH}_{3}\right), 1.67-1.60\left(\mathrm{~m}, 1 \mathrm{H}, \mathrm{CH}_{b} \mathrm{CH}_{3}\right), 1.19\left(\mathrm{t}, 3 \mathrm{H}, \mathrm{J} 7.2 \mathrm{~Hz}, \mathrm{CH}_{3} \mathrm{CH}_{2} \mathrm{O}\right), 0.90(\mathrm{t}$, $3 \mathrm{H}$, J $\left.7.2 \mathrm{~Hz}, \mathrm{CH}_{3} \mathrm{CH}_{2}\right) ;{ }^{13} \mathrm{C}$ NMR (100 MHz, DMSO-d $\left.)_{6}\right), \delta_{\mathrm{C}}(\mathrm{ppm}) 172.0(\mathrm{C}=\mathrm{O}), 167.7(\mathrm{C}=\mathrm{O}), 165.9(\mathrm{C}=\mathrm{O}), 165.8$ 
(C=O), 160,7 (Ar-COH), 134.3 (Aryl-CH), 133.1 (2 × Ar-CH), 124.3 (Ar-C), 116.9 (2 × Ar-CH), 116.8 (Ar-C), 61.0 $\left(\mathrm{OCH}_{2} \mathrm{CH}_{3}\right), 54,0(\mathrm{CHNH}), 43.5\left(\mathrm{CH}_{2} \mathrm{~N}\right), 24.9\left(\mathrm{CH}_{2} \mathrm{CH}_{3}\right), 14.5\left(\mathrm{CH}_{3} \mathrm{CH}_{2} \mathrm{O}\right), 10.6\left(\mathrm{CH}_{3} \mathrm{CH}_{2}\right)$; HRMS (ESI-TOF) $\mathrm{m} / \mathrm{z}$; calculated for $\mathrm{C}_{18} \mathrm{H}_{20} \mathrm{~N}_{2} \mathrm{O}_{6} \mathrm{~S}+\mathrm{H}: 392.1042$, found $\mathrm{M}^{+}+\mathrm{H}: 393.1045$.

Ethyl 2-(2-(5-(furan-2-ylmethylene)-2,4-dioxothiazolidin-3-yl)acetamido)butanoate (13cv) A reaction of furfural $(0.20 \mathrm{ml}, 2.43 \mathrm{mmol})$ and $12 \mathrm{c}(0.70 \mathrm{~g}, 2.43 \mathrm{mmol})$ gave compound $13 \mathrm{cv}$ as a white solid $(0.11 \mathrm{~g} \mathrm{~g}, 11 \%)$; $\mathrm{m} . \mathrm{p}=184-185^{\circ} \mathrm{C} ; \mathrm{IR}\left(\mathrm{KBr} \mathrm{cm}^{-1}\right): 3292,2971,1735,1686,1660,1615,1144 ;{ }^{1} \mathrm{H}$ NMR $\left(400 \mathrm{MHz}, \mathrm{DMSO}-d_{6}\right) \delta_{\mathrm{H}}$ (ppm) $8.69(\mathrm{~d}, 1 \mathrm{H}, J 8.4 \mathrm{~Hz}, \mathrm{NH}), 8.10(\mathrm{~d}, 1 \mathrm{H}, J 1.2 \mathrm{~Hz}, \mathrm{CH}), 7.80(\mathrm{~s}, 1 \mathrm{H}, \operatorname{Aryl}-\mathrm{H}), 7.17(\mathrm{~d}, 1 \mathrm{H}, J 3.6 \mathrm{~Hz}, \mathrm{CH}), 6.77$ (dd, $1 \mathrm{H}, J 3.6 \mathrm{~Hz}$, and J $1.6 \mathrm{~Hz}, \mathrm{CH}$ ), $4.14\left(\mathrm{~s}, 2 \mathrm{H}, \mathrm{CH}_{2} \mathrm{~N}\right.$ ), 4.11 (quint, $1 \mathrm{H}, J 3.6 \mathrm{~Hz}, \mathrm{CHNH}$ ), $4.07(q, 2 \mathrm{H}, J 3.6 \mathrm{~Hz}$, $\left.\mathrm{OCH}_{2} \mathrm{CH}_{3}\right), 1.77-1.72\left(\mathrm{~m}, 1 \mathrm{H}, \mathrm{CH}_{a} \mathrm{CH}_{3}\right)$ 1.71-1.60 (m, $\left.1 \mathrm{H}, \mathrm{CH}_{b} \mathrm{CH}_{3}\right), 1.18\left(\mathrm{t}, 3 \mathrm{H}, \mathrm{J} 7.0 \mathrm{~Hz}, \mathrm{CH}_{3} \mathrm{CH}_{2} \mathrm{O}\right), 0.90(\mathrm{t}, 3 \mathrm{H}, \mathrm{J}$ $\left.7.6 \mathrm{~Hz}, \mathrm{CH}_{3} \mathrm{CH}_{2}\right) ;{ }^{13} \mathrm{C} \mathrm{NMR}\left(100 \mathrm{MHz}\right.$, DMSO- $\left.d_{6}\right), \delta_{c}(\mathrm{ppm}) 172.0(\mathrm{C}=0), 168.2(\mathrm{C}=0), 165.8(\mathrm{C}=0), 165.5(\mathrm{C}=0)$, 149,6 (C), $148.4(\mathrm{CH}), 120.2$ (Aryl-CH), $119.9(\mathrm{CH}), 118.9(\mathrm{C}), 114.1(\mathrm{CH}), 61.0\left(\mathrm{OCH}_{2} \mathrm{CH}_{3}\right), 54,0\left(\mathrm{OCH}_{3}\right), 43.5$ $\left(\mathrm{CH}_{2} \mathrm{~N}\right), 24.9\left(\mathrm{CH}_{2} \mathrm{CH}\right), 14.5\left(\mathrm{CH}_{3} \mathrm{CH}_{2} \mathrm{O}\right), 10.6\left(\mathrm{CH}_{3} \mathrm{CH}_{2}\right)$; HRMS (ESI-TOF) $\mathrm{m} / z$; calculated for $\mathrm{C}_{16} \mathrm{H}_{18} \mathrm{~N}_{2} \mathrm{O}_{6} \mathrm{~S}+\mathrm{H}$ : 367.0886, found $\mathrm{M}^{+}+\mathrm{H}: 367.0889$.

Ethyl (2-(5-benzylidene-2,4-dioxothiazolidin-3-yl)acetyl)valinate (13di). A reaction of benzaldehyde $(0.22 \mathrm{ml}$, $2.08 \mathrm{mmol}$ ) and $12 \mathrm{~d}(0.70 \mathrm{~g}, 2.08 \mathrm{mmol})$ gave compound 13di as a yellow solid (0.26 g, 24\%); m.p = 167-168 ${ }^{\circ} \mathrm{C}$; IR $\left(\mathrm{KBr} \mathrm{cm}^{-1}\right)$ : 3294, 2963, 1734, 1688, 1663, 1608, 1147; ${ }^{1} \mathrm{H}$ NMR (400 MHz, DMSO-d $)_{6} \delta_{H}(p p m) 8.67$ (d, $1 \mathrm{H}, J 8.4 \mathrm{~Hz}, \mathrm{NH}), 7.97(\mathrm{~s}, 1 \mathrm{H}, \mathrm{Aryl}-\mathrm{H}), 7.65(\mathrm{~d}, 2 \mathrm{H}, J 7.2 \mathrm{~Hz}, 2 \times \mathrm{Ar}-\mathrm{H}), 7.58-7.52(\mathrm{~m}, 3 \mathrm{H}, 3 \times \mathrm{Ar}-\mathrm{H}), 4.39(\mathrm{~s}, 2 \mathrm{H}$, $\mathrm{CH}_{2} \mathrm{~N}$ ), 4.18 (quint, $1 \mathrm{H}, J 6.0 \mathrm{~Hz}, \mathrm{CHNH}$ ), 4.12 (q, $2 \mathrm{H}, J 2.4 \mathrm{~Hz}, \mathrm{OCH}_{2} \mathrm{CH}_{3}$ ), 2.06 (septet, $1 \mathrm{H}, J 4.0 \mathrm{~Hz}, \mathrm{CHCH}_{3}$ ), 1.20 (t, $\left.3 \mathrm{H}, J 4.8 \mathrm{~Hz}, \mathrm{CH}_{3} \mathrm{CH}_{2} \mathrm{O}\right), 0.90\left(\mathrm{t}, 6 \mathrm{H}, J 6.4 \mathrm{~Hz},\left(\mathrm{CH}_{3}\right)_{2} \mathrm{CH}\right) ;{ }^{13} \mathrm{C} \mathrm{NMR}\left(100 \mathrm{MHz}, \mathrm{DMSO}-d_{6}\right), \delta_{\mathrm{C}}(\mathrm{ppm}) 171.6(\mathrm{C}=\mathrm{O})$, 167.5 (C=O), 165.9 (C=O), 165.7 (C=O), 133.9 (Aryl-CH), 130.6 (Ar-C), 129.9 (2 × Ar-CH), 128.6, (Ar-C), $128.5(2 \times$ $\mathrm{Ar}-\mathrm{CH}), 121.5(\mathrm{Ar}-\mathrm{C}), 61.4\left(\mathrm{OCH}_{2} \mathrm{CH}_{3}\right), 54.1(\mathrm{CHNH}), 43.6\left(\mathrm{CH}_{2} \mathrm{~N}\right), 30.6\left(\mathrm{CHCH}_{3}\right), 19.3\left(\mathrm{CH}_{3} \mathrm{CH}\right), 18.5\left(\mathrm{CH}_{3} \mathrm{CH}\right), 14.3$ $\left(\mathrm{CH}_{3} \mathrm{CH}_{2} \mathrm{O}\right)$; HRMS (ESI-TOF) $\mathrm{m} / \mathrm{z}$; calculated for $\mathrm{C}_{19} \mathrm{H}_{22} \mathrm{~N}_{2} \mathrm{O}_{5} \mathrm{~S}+\mathrm{H}$ : 391.1249, found $\mathrm{M}^{+}+\mathrm{H}: 391.1251$.

Ethyl (2-(5-(4-methoxybenzylidene)-2,4-dioxothiazolidin-3-yl)acetyl)valinate (13dii). A reaction of $p$ anisaldehyde $(0.40 \mathrm{ml}, 3.30 \mathrm{mmol})$ and $12 \mathrm{~d}(0.70 \mathrm{~g}, 3.30 \mathrm{mmol})$ gave compound 13dii as a yellow solid (0.51 $\mathrm{g}$, $38 \%) ; \mathrm{m} . \mathrm{p}=182-183^{\circ} \mathrm{C}$; IR ( $\left.\mathrm{KBr} \mathrm{cm}^{-1}\right): 3240,2939,1740,1735,1663,1601,1172 ;{ }^{1} \mathrm{H}$ NMR (400 MHz, DMSO-d $)$ $\delta_{\mathrm{H}}(\mathrm{ppm}) 8.66(\mathrm{~d}, 1 \mathrm{H}, J 8.0 \mathrm{~Hz}, \mathrm{NH}), 7.92(\mathrm{~s}, 1 \mathrm{H}, \mathrm{Aryl}-\mathrm{H}), 7.61(\mathrm{~d}, 2 \mathrm{H}, J 8.0 \mathrm{~Hz}, 2 \times \mathrm{Ar}-\mathrm{H}), 7.12(\mathrm{~d}, 2 \mathrm{H}, J 8.0 \mathrm{~Hz}, 2 \times$ $\operatorname{Ar}-\mathrm{H}$ ), $4.18\left(\mathrm{~s}, 2 \mathrm{H}, \mathrm{CH}_{2} \mathrm{~N}\right.$ ), 4.15 (quint, $1 \mathrm{H}, J 3.6 \mathrm{~Hz}, \mathrm{CHNH}$ ), 4.10 (q, $\left.2 \mathrm{H}, J 3.6 \mathrm{~Hz}, \mathrm{OCH}_{2} \mathrm{CH}_{3}\right), 3.89\left(\mathrm{~s}, 3 \mathrm{H}, \mathrm{OCH}_{3}\right)$ 2.05 (septet, $\left.1 \mathrm{H}, J 6.8 \mathrm{~Hz}, \mathrm{CH}\left(\mathrm{CH}_{3}\right)_{2}\right), 1.20\left(\mathrm{t}, 3 \mathrm{H}, J 7.2 \mathrm{~Hz}, \mathrm{CH}_{3} \mathrm{CH}_{2} \mathrm{O}\right), 0.90\left(\mathrm{t}, 6 \mathrm{H}, J 6.4 \mathrm{~Hz},\left(\mathrm{CH}_{3}\right)_{2} \mathrm{CH}\right) ;{ }^{13} \mathrm{C} \mathrm{NMR}$ (100 MHz, DMSO- $\left.d_{6}\right), \delta_{c}(p p m) 171.6(\mathrm{C}=0), 167.5(\mathrm{C}=\mathrm{O}), 165.9(\mathrm{C}=\mathrm{O}), 165.8(\mathrm{C}=\mathrm{O}), 161.7\left(\mathrm{Ar}_{-} \mathrm{COCH}_{3}\right), 133.8$ (Aryl-CH), $132.8(2 \times \mathrm{Ar}-\mathrm{CH}), 125.8,(\mathrm{Ar}-\mathrm{C}), 118.2(\mathrm{Ar}-\mathrm{C}), 115.5(2 \times \mathrm{Ar}-\mathrm{CH}), 61.0\left(\mathrm{OCH}_{2} \mathrm{CH}_{3}\right), 58.0(\mathrm{CHNH}), 56.0$ $\left(\mathrm{OCH}_{3}\right), 43.5\left(\mathrm{CH}_{2} \mathrm{~N}\right), 30.6\left(\mathrm{CH}\left(\mathrm{CH}_{3}\right)_{2}\right), 19.3\left(\mathrm{CH}_{3} \mathrm{CH}\right), 18.5\left(\mathrm{CH}_{3} \mathrm{CH}\right), 14.4\left(\mathrm{CH}_{3} \mathrm{CH}_{2} \mathrm{O}\right)$; HRMS (ESI-TOF) $\mathrm{m} / \mathrm{z}$; calculated for $\mathrm{C}_{20} \mathrm{H}_{24} \mathrm{~N}_{2} \mathrm{O}_{6} \mathrm{~S}+\mathrm{H}: 421.1355$, found $\mathrm{M}^{+}+\mathrm{H}: 421.1352$.

Ethyl (2-(5-(4-hydroxy-3-methoxybenzylidene)-2,4-dioxothiazolidin-3-yl)acetyl)valinate (13diii). A reaction of vanillin $(0.37 \mathrm{~g}, 2.40 \mathrm{mmol})$ and $\mathbf{1 2 d}(0.70 \mathrm{~g}, 2.40 \mathrm{mmol})$ gave compound 13diii as a yellow solid $(0.12 \mathrm{~g}, 12 \%)$; $\mathrm{m} . \mathrm{p}=187-188^{\circ} \mathrm{C} ; \mathrm{IR}\left(\mathrm{KBr} \mathrm{cm}^{-1}\right): 3243,2966,1733,1672,1653,1601,1170 ;{ }^{1} \mathrm{H}$ NMR $\left(400 \mathrm{MHz}, \mathrm{DMSO}-d_{6}\right) \delta_{H}$ (ppm) 10.10 (s, 1H, OH), 8.70 (d, 1H, J $8.4 \mathrm{~Hz}, \mathrm{NH}), 7.93$ (s, 1H, Aryl-H), 7.26 (d, 1H, J 1.6 Hz, Ar-H), 7.18 (dd, $2 \mathrm{H}$, $J_{1} 8.4 \mathrm{~Hz}, J 1.6 \mathrm{~Hz}, \mathrm{Ar}-\mathrm{H}$ ), 7.01 (d. $1 \mathrm{H}, J 8.4 \mathrm{~Hz}, \mathrm{Ar}-\mathrm{H}$ ), $4.22\left(\mathrm{~s}, 2 \mathrm{H}, \mathrm{CH}_{2} \mathrm{~N}\right.$ ), 4.20 (quint, $1 \mathrm{H}, J 3.6 \mathrm{~Hz}, \mathrm{CHNH}$ ), 4.15 (q, $2 \mathrm{H}, J 3.6 \mathrm{~Hz}, \mathrm{OCH}_{2} \mathrm{CH}_{3}$ ), $3.89\left(\mathrm{~s}, 1 \mathrm{H}, \mathrm{OCH}_{3}\right), 2.12$ (septet, $\left.1 \mathrm{H}, J 6.8 \mathrm{~Hz}, \mathrm{CH}\left(\mathrm{CH}_{3}\right)_{2}\right), 1.20(\mathrm{t}, 3 \mathrm{H}, J 6.0 \mathrm{~Hz}$, $\left.\mathrm{CH}_{3} \mathrm{CH}_{2} \mathrm{O}\right), 0.95\left(\mathrm{t}, 6 \mathrm{H}, J 6.4 \mathrm{~Hz},\left(\mathrm{CH}_{3}\right)_{2} \mathrm{CH}\right) ;{ }^{13} \mathrm{C} \mathrm{NMR}\left(100 \mathrm{MHz}, \mathrm{DMSO}-d_{6}\right), \delta_{\mathrm{c}}(\mathrm{ppm}) 171.6(\mathrm{C}=0), 167.6(\mathrm{C}=\mathrm{O})$, $165.9(\mathrm{C}=\mathrm{O}), 165.8(\mathrm{C}=\mathrm{O}), 150.3\left(\mathrm{Ar}-\mathrm{COCH}_{3}\right), 148.5(\mathrm{Ar}-\mathrm{COH}), 134.6(\mathrm{Aryl}-\mathrm{CH}), 124.8(\mathrm{Ar}-\mathrm{C}), 124.7(\mathrm{Ar}-\mathrm{CH}), 117.1$ $(\mathrm{Ar}-\mathrm{C}), 116.7(\mathrm{Ar}-\mathrm{CH}), 114.9(\mathrm{Ar}-\mathrm{CH}), 61.0\left(\mathrm{OCH}_{2} \mathrm{CH}_{3}\right), 58.0(\mathrm{CHNH}), 56.1\left(\mathrm{OCH}_{3}\right), 43.5\left(\mathrm{CH}_{2} \mathrm{~N}\right), 30.6\left(\mathrm{CH}\left(\mathrm{CH}_{3}\right)_{2}\right)$, $19.3\left(\mathrm{CH}_{3} \mathrm{CH}\right), 18.5\left(\mathrm{CH}_{3} \mathrm{CH}\right), 14.35\left(\mathrm{CH}_{3} \mathrm{CH}_{2} \mathrm{O}\right)$; HRMS (ESI-TOF) $\mathrm{m} / z$; calculated for $\mathrm{C}_{20} \mathrm{H}_{24} \mathrm{~N}_{2} \mathrm{O}_{7} \mathrm{~S}+\mathrm{H}: 437.1304$, found $\mathrm{M}^{+}+\mathrm{H}: 437.1307$. 
Ethyl (2-(5-(4-hydroxybenzylidene)-2,4-dioxothiazolidin-3-yl)acetyl)valinate (13div). A reaction of 4hydroxybenzaldehyde $(0.40 \mathrm{ml}, 2.40 \mathrm{mmol})$ and $12 \mathrm{~d}(0.70 \mathrm{~g}, 2.40 \mathrm{mmol})$ gave compound 13 div as a yellow solid (0.034 g, 4\%); m.p = 178-179 ${ }^{\circ} \mathrm{C} ; \mathrm{IR}\left(\mathrm{KBr} \mathrm{cm}{ }^{-1}\right): 3299,2968,1739,1730,1682,1660,1172 ;{ }^{1} \mathrm{H} \mathrm{NMR}(400$ $\left.\mathrm{MHz}, \mathrm{DMSO}-d_{6}\right) \delta_{\mathrm{H}}(\mathrm{ppm}) 10.43(\mathrm{~s}, 1 \mathrm{H}, \mathrm{OH}), 8.65$ (d, $\left.1 \mathrm{H}, J 8.4 \mathrm{~Hz}, \mathrm{NH}\right), 7.86(\mathrm{~s}, 1 \mathrm{H}, \mathrm{Aryl}-\mathrm{H}), 7.53(\mathrm{~d}, 2 \mathrm{H}, J 8.8 \mathrm{~Hz}$, $2 \times \mathrm{Ar}-\mathrm{H}), 6.93(\mathrm{~d}, 2 \mathrm{H}, J 8.4 \mathrm{~Hz}, 2 \times \mathrm{Ar}-\mathrm{H}), 4.19\left(\mathrm{~s}, 2 \mathrm{H}, \mathrm{CH}_{2} \mathrm{~N}\right), 4.16$ (quint, $\left.1 \mathrm{H}, J 3.6 \mathrm{~Hz}, \mathrm{CHNH}\right), 4.08(\mathrm{q}, 2 \mathrm{H}, J 3.6$ $\mathrm{Hz}, \mathrm{OCH}_{2} \mathrm{CH}_{3}$ ), 2.06 (septet, $\left.1 \mathrm{H}, J 6.4 \mathrm{~Hz}, \mathrm{CH}\left(\mathrm{CH}_{3}\right)_{2}\right), 1.21$ (t, 3H, J $\left.7.2 \mathrm{~Hz}, \mathrm{CH}_{3} \mathrm{CH}_{2} \mathrm{O}\right), 0.90(\mathrm{t}, 6 \mathrm{H}, J 6.4 \mathrm{~Hz}$, $\left.\left(\mathrm{CH}_{3}\right)_{2} \mathrm{CH}\right) ;{ }^{13} \mathrm{C}$ NMR (100 MHz, DMSO- $\left.d_{6}\right), \delta_{\mathrm{C}}(\mathrm{ppm}) 171.6(\mathrm{C}=0), 167.6(\mathrm{C}=\mathrm{O}), 166.0(\mathrm{C}=0), 165.9(\mathrm{C}=0), 160.7$ (Ar-COH), 134.3 (Aryl-CH), 133.1 (2x Ar-CH), 124.2 (Ar-C), 116.9 (2 × Ar-CH), $61.0\left(\mathrm{OCH}_{2} \mathrm{CH}_{3}\right), 58.0(\mathrm{CHNH}), 43.5$ $\left(\mathrm{CH}_{2} \mathrm{~N}\right), 30.6\left(\mathrm{CH}\left(\mathrm{CH}_{3}\right)_{2}\right), 19.3\left(\mathrm{CH}_{3} \mathrm{CH}\right), 18.5\left(\mathrm{CH}_{3} \mathrm{CH}\right), 14.4\left(\mathrm{CH}_{3} \mathrm{CH}_{2} \mathrm{O}\right)$; HRMS (ESI-TOF) $\mathrm{m} / \mathrm{z}$; calculated for $\mathrm{C}_{19} \mathrm{H}_{22} \mathrm{~N}_{2} \mathrm{O}_{6} \mathrm{~S}+\mathrm{H}: 407.1199$, found $\mathrm{M}^{+}+\mathrm{H}: 407.1202$.

Ethyl (2-(5-(4-Fluorobenzylidene)-2,4-dioxothiazolidin-3-yl)acetyl)valinate (13dv). A reaction of 4fluorobenzaldehyde $(0.25 \mathrm{ml}, 2.32 \mathrm{mmol})$ and $12 \mathrm{~d}(0.70 \mathrm{~g}, 2.32 \mathrm{mmol})$ in ethanol $(20 \mathrm{ml})$ gave compound $13 \mathrm{dv}$ as a yellow solid $(0.11 \mathrm{~g}, 11 \%) ; \mathrm{m} . \mathrm{p}=216-217^{\circ} \mathrm{C} ; \mathrm{IR}\left(\mathrm{KBr} \mathrm{cm}{ }^{-1}\right): 3283,2961,1736,1686,1662,1614,1149 ;{ }^{1} \mathrm{H}$ NMR (400 MHz, DMSO-d $)_{6} \delta_{H}(p p m) 8.65(\mathrm{~d}, 1 \mathrm{H}, J 8.4 \mathrm{~Hz}, \mathrm{NH}), 7.99\left(\mathrm{~s}, 1 \mathrm{H}\right.$, Aryl-H), $7.74\left(\mathrm{dd}, 2 \mathrm{H},{ }^{4} J_{F H} 8.4 \mathrm{~Hz}, J_{H H}\right.$ $5.6 \mathrm{~Hz}, 2 \times \mathrm{Ar}-\mathrm{H}), 7.42\left(\mathrm{dd}, 2 \mathrm{H},{ }^{3} J_{F H} 17.6 \mathrm{~Hz}, J_{H H} 8.8 \mathrm{~Hz}, 2 \times \mathrm{Ar}-\mathrm{H}\right.$ ), $4.17\left(\mathrm{~s}, 2 \mathrm{H}, \mathrm{CH}_{2} \mathrm{~N}\right.$ ), 4.15 (quint, $1 \mathrm{H}, J 3.6 \mathrm{~Hz}$, $\mathrm{CHNH}$ ), 4.10 (q, 2H, J $3.6 \mathrm{~Hz}, \mathrm{OCH}_{2} \mathrm{CH}_{3}$ ), 2.05 (septet, $\left.1 \mathrm{H}, J 6.8 \mathrm{~Hz}, \mathrm{CH}\left(\mathrm{CH}_{3}\right)_{2}\right), 1.20\left(\mathrm{t}, 3 \mathrm{H}, J 7.2 \mathrm{~Hz}, \mathrm{CH}_{3} \mathrm{CH}_{2} \mathrm{O}\right.$ ), $0.90\left(\mathrm{t}, 6 \mathrm{H}, J 6.4 \mathrm{~Hz}, \mathrm{CH}_{3} \mathrm{CH}\right) ;{ }^{19} \mathrm{~F} \mathrm{NMR}(376.5 \mathrm{MHz} \text {, DMSO-d })_{6} \delta_{\mathrm{F}}(\mathrm{ppm})-108.02-(-108.36)(\mathrm{m}, 1 \mathrm{~F}, \mathrm{Ar}-\mathrm{F}) ;{ }^{13} \mathrm{C} \mathrm{NMR}$ (100 MHz, DMSO- $\left.d_{6}\right), \delta_{c}(\mathrm{ppm}) 171.6(\mathrm{C}=0), 167.3(\mathrm{C}=0), 165.8(\mathrm{C}=0), 165.6(\mathrm{C}=0), 163.5(\mathrm{~d}, 1 \mathrm{~J} 250.0 \mathrm{~Hz}, \mathrm{Ar}-$ CF), 133.2 (d, ${ }^{3}$ J $9.0 \mathrm{~Hz}, 2 \times \mathrm{Ar}-\mathrm{CH}$ ), 132.8 (Aryl-CH), 130.0 (d, 4 J $3.0 \mathrm{~Hz}, \mathrm{Ar}-\mathrm{C}$ ), 121.2 (Ar-C), 117.1 (d, ${ }^{2}$ J $22.0 \mathrm{~Hz}, 2$ $\times \mathrm{Ar}-\mathrm{CH}), 61.0\left(\mathrm{OCH}_{2} \mathrm{CH}_{3}\right), 58.1(\mathrm{CHNH}), 43.6\left(\mathrm{CH}_{2} \mathrm{~N}\right), 30.6\left(\mathrm{CH}\left(\mathrm{CH}_{3}\right)_{2}\right), 19.3\left(\mathrm{CH}{ }_{3} \mathrm{CH}\right), 18.5\left(\mathrm{CH}_{3} \mathrm{CH}\right), 14.5$ $\left(\mathrm{CH}_{3} \mathrm{CH}_{2} \mathrm{O}\right)$; HRMS (ESI-TOF) $\mathrm{m} / z$; calculated for $\mathrm{C}_{19} \mathrm{H}_{21} \mathrm{FN}_{2} \mathrm{O}_{5} \mathrm{~S}+\mathrm{H}: 409.1155$, found $\mathrm{M}^{+}+\mathrm{H}: 409.1158$.

Ethyl (2-(5-(4-methoxybenzylidene)-2,4-dioxothiazolidin-3-yl)acetyl)norvalinate (13ei). A reaction of $p$ anisaldehyde $(0.40 \mathrm{ml}, 3.30 \mathrm{mmol})$ and $12 \mathrm{e}(0.70 \mathrm{~g}, 3.30 \mathrm{mmol})$ gave compound 13ei as a white solid (0.22 $\mathrm{g}$, $17 \%) ; \mathrm{m} . \mathrm{p}=204-205^{\circ} \mathrm{C} ; \mathrm{IR}\left(\mathrm{KBr} \mathrm{cm}^{-1}\right): 3303,2960,1734,1687,1663,1593,1183 ;{ }^{1} \mathrm{H} \mathrm{NMR}(400 \mathrm{MHz}$, DMSO$\left.d_{6}\right) \delta_{\mathrm{H}}(\mathrm{ppm}) 8.72(\mathrm{~d}, 1 \mathrm{H}, J 7.6 \mathrm{~Hz}, \mathrm{NH}), 7.91(\mathrm{~s}, 1 \mathrm{H}, \mathrm{Aryl}-\mathrm{H}), 7.61(\mathrm{~d}, 2 \mathrm{H}, J 8.8 \mathrm{~Hz}, 2 \times \mathrm{Ar}-\mathrm{H}), 7.12(\mathrm{~d}, 2 \mathrm{H}, J 8.8 \mathrm{~Hz}$, $2 \times \mathrm{Ar}-\mathrm{H}$ ), $4.32\left(\mathrm{~s}, 2 \mathrm{H}, \mathrm{CH}_{2} \mathrm{~N}\right.$ ), 4.21 (quint, $\left.1 \mathrm{H}, J 5.2 \mathrm{~Hz}, \mathrm{CHNH}\right), 4.09\left(\mathrm{q}, 2 \mathrm{H}, J 3.6 \mathrm{~Hz}, \mathrm{OCH}_{2} \mathrm{CH}_{3}\right), 3.83(\mathrm{~s}, 3 \mathrm{H}$, $\left.\mathrm{OCH}_{3}\right), 1.66-1.60\left(\mathrm{~m}, 2 \mathrm{H}, \mathrm{CH}_{2} \mathrm{CH}_{3}\right), 1.34-1.28\left(\mathrm{~m}, 2 \mathrm{H}, \mathrm{CH}_{2} \mathrm{CH}\right), 1.18\left(\mathrm{t}, 3 \mathrm{H}, J 6.8 \mathrm{~Hz}, \mathrm{CH}_{3} \mathrm{CH}_{2} \mathrm{O}\right), 0.89(\mathrm{t}, 3 \mathrm{H}, J 7.8$ $\left.\mathrm{Hz}, \mathrm{CH}_{3} \mathrm{CH}_{2}\right) ;{ }^{13} \mathrm{C}$ NMR (100 MHz, DMSO- $\left.d_{6}\right), \delta_{\mathrm{C}}(\mathrm{ppm}) 172.3(\mathrm{C}=0), 167.6(\mathrm{C}=0), 165.8(2 \times \mathrm{C}=0), 161.7(\mathrm{Ar}-$ $\left.\mathrm{COCH}_{3}\right), 133.9$ (Aryl-CH), $132.8(2 \times \mathrm{Ar}-\mathrm{CH}), 125.8(\mathrm{Ar}-\mathrm{C}), 118.2(\mathrm{Ar}-\mathrm{C}), 115.5(2 \times \mathrm{Ar}-\mathrm{CH}), 61.0\left(\mathrm{OCH}_{2} \mathrm{CH}_{3}\right), 55.9$ $\left(\mathrm{OCH}_{3}\right), 52.4(\mathrm{CHNH}), 43.5\left(\mathrm{CH}_{2} \mathrm{~N}\right), 33.5\left(\mathrm{CH}_{2} \mathrm{CH}\right), 18.9\left(\mathrm{CH}_{2} \mathrm{CH}_{3}\right), 14.5\left(\mathrm{CH}_{3} \mathrm{CH}_{2} \mathrm{O}\right), 13.9\left(\mathrm{CH}_{3} \mathrm{CH}_{2}\right)$; HRMS (ESI-TOF) $\mathrm{m} / \mathrm{z}$; calculated for $\mathrm{C}_{20} \mathrm{H}_{24} \mathrm{~N}_{2} \mathrm{O}_{6} \mathrm{~S}+\mathrm{H}: 421.1355$, found $\mathrm{M}^{+}+\mathrm{H}: 421.1358$.

Ethyl (2-(5-(4-methylbenzylidene)-2,4-dioxothiazolidin-3-yl)acetyl)norvalinate (13eii). A reaction of $p$ tolualdehyde $(0.29 \mathrm{ml}, 2.32 \mathrm{mmol})$ and $12 \mathrm{e}(0.70 \mathrm{~g}, 2.32 \mathrm{mmol})$ gave compound 13eii as a white solid (0.12 $\mathrm{g}$, $13 \%) ; \mathrm{m} . \mathrm{p}=196-197^{\circ} \mathrm{C}$; IR $\left(\mathrm{KBr} \mathrm{cm}^{-1}\right): 3241,2960,1734,1689,1661,1599,1149 ;{ }^{1} \mathrm{H}$ NMR $\left(400 \mathrm{MHz}, \mathrm{DMSO}-d_{6}\right)$ $\delta_{\mathrm{H}}(\mathrm{ppm}) 8.72(\mathrm{~d}, 1 \mathrm{H}, J 7.6 \mathrm{~Hz}, \mathrm{NH}), 7.91(\mathrm{~s}, 1 \mathrm{H}, \operatorname{Aryl}-\mathrm{H}), 7.54(\mathrm{~d}, 2 \mathrm{H}, J 8.0 \mathrm{~Hz}, 2 \times \mathrm{Ar}-\mathrm{H}), 7.37(\mathrm{~d}, 2 \mathrm{H}, J 8.0 \mathrm{~Hz}, 2 \times$ $\operatorname{Ar}-\mathrm{H}$ ), $4.32\left(\mathrm{~s}, 2 \mathrm{H}, \mathrm{CH}_{2} \mathrm{~N}\right.$ ), 4.21 (quint, $1 \mathrm{H}, J 5.2 \mathrm{~Hz}, \mathrm{CHNH}$ ), 4.09 (q, $\left.2 \mathrm{H}, J 3.6 \mathrm{~Hz}, \mathrm{OCH}_{2} \mathrm{CH}_{3}\right), 2.37\left(\mathrm{~s}, 3 \mathrm{H}, \mathrm{Ar}-\mathrm{CH}_{3}\right)$, 1.69-1.57 (m, 2H, CH$\left.{ }_{2} \mathrm{CH}_{3}\right), 1.37-1.34\left(\mathrm{~m}, 2 \mathrm{H}, \mathrm{CH}_{2} \mathrm{CH}\right), 1.18\left(\mathrm{t}, 3 \mathrm{H}, J 6.8 \mathrm{~Hz}, \mathrm{CH}_{3} \mathrm{CH}_{2} \mathrm{O}\right), 0.89(\mathrm{t}, 3 \mathrm{H}, J 7.8 \mathrm{~Hz}$, $\left.\mathrm{CH}_{3} \mathrm{CH}_{2}\right) ;{ }^{13} \mathrm{C}$ NMR $\left(100 \mathrm{MHz}, \mathrm{DMSO}-d_{6}\right), \delta_{\mathrm{C}}(\mathrm{ppm}) 172.3(\mathrm{C}=\mathrm{O}), 167.6(\mathrm{C}=\mathrm{O}), 165.8(2 \times \mathrm{C}=0), 141.7\left(\mathrm{Ar}-\mathrm{CCH}_{3}\right)$, 133.9 (Aryl-CH), 130.7 (2 $\times \mathrm{Ar}-\mathrm{CH}), 130.3(2 \times \mathrm{Ar}-\mathrm{CH}), 120.3(\mathrm{Ar}-\mathrm{C}), 61.0\left(\mathrm{OCH}_{2} \mathrm{CH}_{3}\right), 52.4(\mathrm{CHNH}), 43.5\left(\mathrm{CH}_{2} \mathrm{~N}\right)$, $33.5\left(\mathrm{CH}_{2} \mathrm{CH}\right), 21.6\left(\mathrm{Ar}-\mathrm{CH}_{3}\right), 18.9\left(\mathrm{CH}_{2} \mathrm{CH}_{3}\right), 14.5\left(\mathrm{CH}_{3} \mathrm{CH}_{2} \mathrm{O}\right), 13.9\left(\mathrm{CH}_{3} \mathrm{CH}_{2}\right) ;$ HRMS (ESI-TOF) $\mathrm{m} / \mathrm{z}$; calculated for $\mathrm{C}_{20} \mathrm{H}_{24} \mathrm{~N}_{2} \mathrm{O}_{5} \mathrm{~S}+\mathrm{H}: 405.1406$, found $\mathrm{M}^{+}+\mathrm{H}: 405.1409$.

Ethyl (2-(5-(4-hydroxybenzylidene)-2,4-dioxothiazolidin-3-yl)acetyl)norvalinate (13eiii). A reaction of 4hydroxybenzaldehyde $(0.30 \mathrm{~g}, 2.42 \mathrm{mmol})$ and $12 \mathrm{e}(0.70 \mathrm{~g}, 2.42 \mathrm{mmol})$ gave compound 13eiii as a yellow solid $(0.24 \mathrm{~g}, 26 \%) ; \mathrm{m} . \mathrm{p}=125-126{ }^{\circ} \mathrm{C} ; \mathrm{IR}\left(\mathrm{KBr} \mathrm{cm}^{-1}\right): 3308,2989,1730,1668,1631,1601,1153 ;{ }^{1} \mathrm{H} \mathrm{NMR}(400 \mathrm{MHz}$, 
DMSO-d $)_{6} \delta_{H}(p p m) 10.36(\mathrm{~s}, 1 \mathrm{H}, \mathrm{OH}), 8.66(\mathrm{~d}, 1 \mathrm{H}, J 7.6 \mathrm{~Hz}, \mathrm{NH}), 7.86(\mathrm{~s}, 1 \mathrm{H}$, Aryl-H), $7.51(\mathrm{~d}, 2 \mathrm{H}, J 8.4 \mathrm{~Hz}, 2 \times$ $\operatorname{Ar}-\mathrm{H}$ ), $6.93(\mathrm{~d}, 2 \mathrm{H}, J 8.8 \mathrm{~Hz}, 2 \times \mathrm{Ar}-\mathrm{H}), 4.25\left(\mathrm{~s}, 2 \mathrm{H}, \mathrm{CH}_{2} \mathrm{~N}\right.$ ), 4.22 (quint, $\left.1 \mathrm{H}, J 5.2 \mathrm{~Hz}, \mathrm{CHNH}\right), 4.10(\mathrm{q}, 2 \mathrm{H}, J 3.6 \mathrm{~Hz}$, $\left.\mathrm{OCH}_{2} \mathrm{CH}_{3}\right), 1.69-1.59\left(\mathrm{~m}, 2 \mathrm{H}, \mathrm{CH}_{2} \mathrm{CH}_{3}\right), 1.36-1.30\left(\mathrm{~m}, 2 \mathrm{H}, \mathrm{CH}_{2} \mathrm{CH}\right), 1.18\left(\mathrm{t}, 3 \mathrm{H}, J 6.8 \mathrm{~Hz}, \mathrm{CH}_{3} \mathrm{CH}_{2} \mathrm{O}\right), 0.88(\mathrm{t}, 3 \mathrm{H}, J$ $\left.7.2 \mathrm{~Hz}, \mathrm{CH}_{3} \mathrm{CH}_{2}\right) ;{ }^{13} \mathrm{C}$ NMR (100 MHz, DMSO-d $)$, $\delta_{c}(\mathrm{ppm}) 172.2(\mathrm{C}=0), 167.6(\mathrm{C}=0), 165.9(\mathrm{C}=0), 165.8(\mathrm{C}=0)$, 160.7 (Ar- $\mathrm{COH}), 134.3$ (Aryl-CH), 133.1 (2 × Ar-CH), $124.3(\mathrm{Ar}-\mathrm{C}), 117.0(\mathrm{Ar}-\mathrm{C}), 116.9(2 \times \mathrm{Ar}-\mathrm{CH}), 61.0$ $\left(\mathrm{OCH}_{2} \mathrm{CH}_{3}\right), 52.4(\mathrm{CHNH}), 43.6\left(\mathrm{CH}_{2} \mathrm{~N}\right), 33.6\left(\mathrm{CH}_{2} \mathrm{CH}\right), 18.9\left(\mathrm{CH}_{2} \mathrm{CH}_{3}\right), 14.5\left(\mathrm{CH}_{3} \mathrm{CH}_{2} \mathrm{O}\right), 13.9\left(\mathrm{CH}_{3} \mathrm{CH}_{2}\right)$; HRMS (ESITOF) $m / z$; calculated for $\mathrm{C}_{19} \mathrm{H}_{22} \mathrm{~N}_{2} \mathrm{O}_{6} \mathrm{~S}+\mathrm{H}: 407.1199$, found $\mathrm{M}^{+}+\mathrm{H}: 407.1202$.

Ethyl (2-(5-(4-fluorobenzylidene)-2,4-dioxothiazolidin-3-yl)acetyl)norvalinate (13eiv). A reaction of 4fluorobenzaldehyde $(0.16 \mathrm{ml}, 1.32 \mathrm{mmol})$ and $12 \mathrm{e}(0.40 \mathrm{~g}, 1.32 \mathrm{mmol})$ gave compound 13eiv as a yellow solid $(0.11 \mathrm{~g}, 21 \%) ; \mathrm{m} . \mathrm{p}=240-24{ }^{\circ} \mathrm{C}$; IR $\left(\mathrm{KBr} \mathrm{cm}^{-1}\right): 3285,2964,1735,1686,1657,1609,1147 ;{ }^{1} \mathrm{H} \mathrm{NMR}(400 \mathrm{MHz}$, DMSO- $\left.d_{6}\right) \delta_{H}(\mathrm{ppm}) 8.72(\mathrm{~d}, 1 \mathrm{H}, J 7.6 \mathrm{~Hz}, \mathrm{NH}), 7.99(\mathrm{~s}, 1 \mathrm{H}, \mathrm{Aryl}-\mathrm{H}), 7.74\left(\mathrm{dd}, 2 \mathrm{H},{ }^{4} J_{F H} 8.8 \mathrm{~Hz}, J_{H H} 5.6 \mathrm{~Hz}, 2 \times \mathrm{Ar}-\mathrm{H}\right)$, $7.42\left(\mathrm{dd}, 2 \mathrm{H}, 3^{3} \mathrm{JH}_{\mathrm{FH}} 17.2 \mathrm{~Hz}, J_{H H} 8.4 \mathrm{~Hz}, 2 \times \mathrm{Ar}-\mathrm{H}\right.$ ), $4.23\left(\mathrm{~s}, 2 \mathrm{H}, \mathrm{CH}_{2} \mathrm{~N}\right.$ ), 4.13 (quint, $1 \mathrm{H}, J 3.6 \mathrm{~Hz}, \mathrm{CHNH}$ ), 4.09 (q, $2 \mathrm{H}$, J $\left.5.2 \mathrm{~Hz}, \mathrm{OCH}_{2} \mathrm{CH}_{3}\right), 1.69-1.59\left(\mathrm{~m}, 2 \mathrm{H}, \mathrm{CH}_{2} \mathrm{CH}_{3}\right), 1.36-1.30\left(\mathrm{~m} 2 \mathrm{H}, \mathrm{CH}_{2} \mathrm{CH}\right), 1.88$ (t, 3H, J 7.2 Hz, $\left.\mathrm{CH}_{3} \mathrm{CH}_{2} \mathrm{O}\right), 0.88(\mathrm{t}$, $3 \mathrm{H}$, J $\left.7.2 \mathrm{~Hz}, \mathrm{CH}_{3} \mathrm{CH}_{2}\right) ;{ }^{19} \mathrm{~F}$ NMR $(376.5 \mathrm{MHz} \text {, DMSO-d })_{6} \delta_{\mathrm{F}}(\mathrm{ppm})-108.28-(-108.36)(\mathrm{m}, 1 \mathrm{~F}, \operatorname{Ar}-\mathrm{F}) ;{ }^{13} \mathrm{C} \mathrm{NMR}(100$ $\left.\mathrm{MHz}, \mathrm{DMSO}-d_{6}\right), \delta_{c}(\mathrm{ppm}) 172.2(\mathrm{C}=0), 167.3(\mathrm{C}=0), 165.7(\mathrm{C}=0), 165.6(\mathrm{C}=0), 163.6$ (d, 1 J 250.0 Hz, Ar-CF), 133.1 (d, ${ }^{3}$ J $9.0 \mathrm{~Hz}, 2 \times \mathrm{Ar}-\mathrm{CH}$ ), 132.8 (Aryl-CH), 130.0 (d, ${ }^{4}$ J $\left.3.0 \mathrm{~Hz}, \mathrm{Ar}-\mathrm{C}\right), 121.2$ (Ar-C), 117.1 (d, ${ }^{2}$ J $22.0 \mathrm{~Hz}, 2 \times$ $\operatorname{Ar}-\mathrm{CH}), 61.0\left(\mathrm{OCH}_{2} \mathrm{CH}_{3}\right), 52.4(\mathrm{CHNH}), 43.6\left(\mathrm{CH}_{2} \mathrm{~N}\right)$, $33.9\left(\mathrm{CH}_{2} \mathrm{CH}\right), 18.9\left(\mathrm{CH}_{2} \mathrm{CH}_{3}\right), 14.5\left(\mathrm{CH}_{3} \mathrm{CH}_{2} \mathrm{O}\right), 13.9\left(\mathrm{CH}_{3} \mathrm{CH}_{2}\right)$; HRMS (ESI-TOF) $m / z$; calculated for $\mathrm{C}_{19} \mathrm{H}_{21} \mathrm{FN}_{2} \mathrm{O}_{5} \mathrm{~S}+\mathrm{H}$ : 409.1155, found $\mathrm{M}^{+}+\mathrm{H}: 409.1158$.

Ethyl (2-(5-(furan-2-ylmethylene)-2,4-dioxothiazolidin-3-yl)acetyl)norvalinate (13ev). A reaction of furfural $(0.20 \mathrm{ml}, 2.42 \mathrm{mmol})$ and $12 \mathrm{e}(0.70 \mathrm{~g}, 2.42 \mathrm{mmol})$ gave compound $13 \mathrm{ev}$ as a brown solid $(0.61 \mathrm{~g}, 65 \%) ; \mathrm{m} . \mathrm{p} \mathrm{=}$ $181-182{ }^{\circ} \mathrm{C}$; IR $\left(\mathrm{KBr} \mathrm{cm}{ }^{-1}\right): 3285,2959,1735,1684,1662,1615,1147 ;{ }^{1} \mathrm{H}$ NMR $\left(400 \mathrm{MHz}, \mathrm{DMSO}-d_{6}\right) \delta_{\mathrm{H}}(\mathrm{ppm})$ 8.65 (dd, $\left.1 \mathrm{H}, J_{1} 21.2 \mathrm{~Hz}, J_{2} 7.6 \mathrm{~Hz}, \mathrm{Ar}-\mathrm{H}\right), 8.10$ (s, 1H, NH), 7.79 (s, 1H, Aryl-H), 7.17 (d, $\left.1 \mathrm{H}, J 3.6 \mathrm{~Hz}, \mathrm{Ar}-\mathrm{H}\right), 6.78$ (t, $1 \mathrm{H}, J 1.6 \mathrm{~Hz}, \mathrm{Ar}-\mathrm{H}$ ), $4.36\left(\mathrm{~s}, 2 \mathrm{H}, \mathrm{CH}_{2} \mathrm{~N}\right.$ ), 4.23 (quint, $1 \mathrm{H}, J 5.6 \mathrm{~Hz}, \mathrm{CHNH}$ ), 4.11 (q, $\left.2 \mathrm{H}, J 3.6 \mathrm{~Hz}, \mathrm{OCH}_{2} \mathrm{CH}_{3}\right), 1.56-$ $1.48\left(\mathrm{~m}, 2 \mathrm{H}, \mathrm{CH}_{2} \mathrm{CH}_{3}\right), 1.36-1.32\left(\mathrm{~m}, 2 \mathrm{H}, \mathrm{CH}_{2} \mathrm{CH}\right), 1.20\left(\mathrm{t}, 3 \mathrm{H}, J 6.8 \mathrm{~Hz}, \mathrm{CH}_{3} \mathrm{CH}_{2} \mathrm{O}\right), 0.88\left(\mathrm{t}, 3 \mathrm{H}, J 7.2 \mathrm{~Hz}, \mathrm{CH}_{3} \mathrm{CH}_{2}\right)$; ${ }^{13} \mathrm{C}$ NMR $\left(100 \mathrm{MHz}, \mathrm{DMSO}-d_{6}\right), \delta_{c}(\mathrm{ppm}) 172.2(\mathrm{C}=0), 168.1(\mathrm{C}=0), 165.9(\mathrm{C}=0), 165.7(\mathrm{C}=0), 149.6(\mathrm{Ar}-\mathrm{C})$, 148.4 Ar-CH), 120.2 (Aryl-CH), 119.9 (Ar-CH), $118.2(\mathrm{Ar}-\mathrm{C}), 114.1(\mathrm{Ar}-\mathrm{CH}), 60.9\left(\mathrm{OCH}_{2} \mathrm{CH}_{3}\right), 58.1(\mathrm{CHNH}), 43.5$ $\left(\mathrm{CH}_{2} \mathrm{~N}\right), 33.6\left(\mathrm{CH}_{2} \mathrm{CH}\right), 18.9\left(\mathrm{CH}_{2} \mathrm{CH}_{3}\right), 14.5\left(\mathrm{CH}_{3} \mathrm{CH}_{2} \mathrm{O}\right), 13.9\left(\mathrm{CH}_{3} \mathrm{CH}_{2}\right)$; HRMS (ESI-TOF) $\mathrm{m} / z$; calculated for $\mathrm{C}_{17} \mathrm{H}_{20} \mathrm{~N}_{2} \mathrm{O}_{6} \mathrm{~S}+\mathrm{H}: 381,1042$, found $\mathrm{M}^{+}+\mathrm{H}: 381.1045$.

\section{Acknowledgements}

The authors would like to thank the National Research Foundation (NRF) of South Africa and the Sasol Inzalo Foundation for the scholarships offered to Mr NR Tshiluka and the THUTHUKA research funds for sponsoring this research.

\section{Supplementary Material}

Supplementary data $\left({ }^{1} \mathrm{H} N M R,{ }^{13} \mathrm{C} N M R,{ }^{19} \mathrm{~F}\right.$ NMR and IR spectra of all the products) associated with this paper can be found in the online version.

\section{References}

1. Bansal, G.; Thanikachalam, P. V.; Maurya, R. K.; Chawla, P.; Ramamurthy, S. J. Adv. Res. 2020, 23, $163-205$. https://doi.org/10.1016/j.jare.2020.01.008 
2. Datar, P. A.; Aher, S. B. J. Saudi Chem. Soc. 2016, 20, S196-S201. https://doi.org/10.1016/j.jscs.2012.10.010

3. Bozdağ-Dündar, O.; Evcimen, N. D.; Ceylan-Ünlüsoy, M.; Ertan, R.; Sarikaya, M. Med. Chem. Res. 2007, 16, 39-47.

https://doi.org/10.1007/s00044-007-9008-9

4. Aneja, D. K., Lohan, P., Arora, S., Sharma, C., Aneja, K.R., Prakash, O. Org. Med. Chem. Lett. 2011, 1, 15. https://doi.org/10.1186/2191-2858-1-15

5. Patil, V.; Tilekar, K.; Mehendale-Munj, S.; Mohan, R.; Ramaa, C. S. Eur. J. Med. Chem. 2010, 45, 4539-4544. https://doi.org/10.1016/j.ejmech.2010.07.014

6. Rekha, S.; Shantharam, U.; Vineet, C. Int. Res. J. Pharm. 2011, 2, 81-84 ().

7. Sharma, R. K., Younis, Y., Mugumbate, G., Njoroge, M., Gut, J., Rosenthal, P. J., Chibale, K. Eur. J. Med. Chem. 2015, 90, 507-518. https://doi.org/10.1016/i.ejmech.2014.11.061

8. Burrows, J. N.; Burlot, E.; Campo, B.; Cherbuin, S.; Jeanneret, S.; Leroy, D.; Spangenberg, T.; Waterson, D.; Wells, T. N. C.; Willis, P. Parasitology 2014, 141, 128-139. https://doi.org/10.1017/S0031182013000826

9. Alagawadi, K. R. \& Alegaon, S. G. Arab. J. Chem. 2011, 4, 465-472. https://doi.org/10.1016/j.arabjc.2010.07.012

10. Kumar, H.; Deep, A.; Marwaha, R. K. BMC Chem. 2020, 14, 1-15. https://doi.org/10.1186/s13065-020-00678-2

11. Kumar, B. R. P.; Kumar, S. S.; Viral, P.; Wadhwani, A.; Vadivelan, R.; Satish Kumar, M. N.; Nanjan, M. J. Med. Chem. Res. 2012, 21, 2689-2701.

https://doi.org/10.1007/s00044-011-9792-0

12. Ali, A. M.; Saber, G. E.; Mahfouz, N. M.; El-Gendy, M. A.; Radwant, A. A.; El-Hamid, M. A. Arch. Pharmacal Res. 2007, 30, 1186-1204. https://doi.org/10.1007/BF02980259

13. Kumar, B. R. P.; Soni, M.; Kumar, S.; Singh, K; Patil, M; Nasir Baig, R. B.; Adhikary, L. Eur. J. Med. Chem. 2011, 46, 835-844. https://doi.org/10.1016/j.ejmech.2010.12.019

14. Aladzheva, I. M.; Bykhovskaya, O. V.; Petrovskii, P. V.; Mastryukova, T. A. Russian Chem. Bull. 2007, 56, 2456-2459.

https://doi.org/10.1007/s11172-007-0390-8

15. Yuan, F.; Hou, Z.; Pramanick, P. K.; Yao, B. Site-selective modification of $\alpha$-amino acids and oligopeptides via native amine-directed $\mathrm{\gamma}-\mathrm{C}(\mathrm{sp} 3)-\mathrm{H}$ arylation. Org. Lett. 2019, 21, 9381-9385. https://doi.org/10.1021/acs.orglett.9b03607

16. Rachedi, K. O.; Ouk, T. S.; Bahadi, R.; Bouzina, A.; Djouad, S. E.; Bechlem, K.; Zerrouki, R.; Hadda, T. B.; Almalki, F.; Berredjem, M. J. Mol. Struct. 2019, 1197, 196-203. https://doi.org/10.1016/i.molstruc.2019.07.053 